\title{
On the Buratti-Horak-Rosa Conjecture about Hamiltonian Paths in Complete Graphs
}

\author{
Anita Pasotti \\ DICATAM - Sez. Matematica \\ Università degli Studi di Brescia \\ Via Branze 43 \\ I-25123 Brescia, Italy \\ anita.pasotti@unibs.it
}

\author{
Marco Antonio Pellegrini \\ Dipartimento di Matematica e Fisica \\ Università Cattolica del Sacro Cuore \\ Via Musei 41 \\ I-25121 Brescia, Italy \\ marcoantonio.pellegrini@unicatt.it
}

Submitted: Nov 12, 2013; Accepted: Apr 24, 2014; Published: May 13, 2014

Mathematics Subject Classifications: 05C38

\begin{abstract}
In this paper we investigate a problem proposed by Marco Buratti, Peter Horak and Alex Rosa (denoted by BHR-problem) concerning Hamiltonian paths in the complete graph with prescribed edge-lengths. In particular we solve $\operatorname{BHR}\left(\left\{1^{a}, 2^{b}\right.\right.$, $\left.\left.t^{c}\right\}\right)$ for any even integer $t \geqslant 4$, provided that $a+b \geqslant t-1$. Furthermore, for $t=4,6,8$ we present a complete solution of $\operatorname{BHR}\left(\left\{1^{a}, 2^{b}, t^{c}\right\}\right)$ for any positive integer $a, b, c$.
\end{abstract}

Keywords: Hamiltonian path; complete graph; edge-length

\section{Introduction}

Throughout this paper $K_{v}$ will denote the complete graph on $\{0,1, \ldots, v-1\}$ for any positive integer $v$. For the basic terminology on graphs we refer to [9]. Following [6], we define the length $\ell(x, y)$ of an edge $[x, y]$ of $K_{v}$ as

$$
\ell(x, y)=\min (|x-y|, v-|x-y|) .
$$

If $\Gamma$ is any subgraph of $K_{v}$, then the list of edge-lengths of $\Gamma$ is the multiset $\ell(\Gamma)$ of the lengths (taken with their respective multiplicities) of all the edges of $\Gamma$. For our convenience, if a list $L$ consists of $a_{1} 1^{\prime} \mathrm{s}, a_{2} 2^{\prime} \mathrm{s}, \ldots, a_{t} t^{\prime} \mathrm{s}$, we will write $L=\left\{1^{a_{1}}, 2^{a_{2}}, \ldots, t^{a_{t}}\right\}$.

The following conjecture $[3,8]$ is due to Marco Buratti (2007, communication to Alex Rosa). 
Conjecture 1 (Buratti). For any prime $p=2 n+1$ and any multiset $L$ of $2 n$ positive integers not exceeding $n$, there exists a Hamiltonian path $H$ of $K_{p}$ with $\ell(H)=L$.

The conjecture is almost trivially true in the case that $L$ has just one edge-length, but, in general, the problem seems to be very difficult. The case of exactly two distinct edge-lengths has been solved independently in [5, 6] and Mariusz Meszka checked that the conjecture is true for all primes $p \leqslant 23$ by computer. Some general results on the conjecture can be found in [6], in particular that it is true when there is an edge-length occurring "sufficiently many times" in $L$.

In [6] Peter Horak and Alex Rosa generalized Buratti's conjecture. Such a generalization has been restated in an easier form in [7], as follows.

Conjecture 2 (Horak and Rosa). Let $L$ be a list of $v-1$ positive integers not exceeding $\left\lfloor\frac{v}{2}\right\rfloor$. Then there exists a Hamiltonian path $H$ of $K_{v}$ such that $\ell(H)=L$ if, and only if, the following condition holds:

\section{for any divisor $d$ of $v$, the number of multiples of $d$} appearing in $L$ does not exceed $v-d$.

Following [7], by $\operatorname{BHR}(L)$ we will denote the above conjecture for a given list $L$. The case of exactly three distinct edge-lengths has been solved when these lengths are 1,2,3 in [4] and when they are 1,2,5 or 1,3,5 or 2,3,5 in [7]. More in general, in [7] we proved that $\operatorname{BHR}\left(\left\{1^{a}, 2^{b}, 3^{c}, 5^{d}\right\}\right)$ holds for all integers $a, b, c, d \geqslant 0$ finding, in such a way, the first set $S$ of size four for which we can say that $\operatorname{BHR}(L)$ is true when the underlying-set of the list $L$ is $S$.

In the same paper we have also shown how this problem is related to the existence of cyclic graph decompositions. In detail, we proved that $\operatorname{BHR}(L)$ can be reformulated as follows.

Conjecture 3. A Cayley multigraph $\operatorname{Cay}\left[\mathbb{Z}_{v}: \Lambda\right]$ admits a cyclic decomposition into Hamiltonian paths if and only if $\Lambda=L \cup-L$ with $L$ satisfying condition (1).

For reader convenience we recall the definition of a Cayley multigraph, see [1, 2]. Given an additive group $G$ and a list $\Lambda \subseteq G \backslash\{0\}$ such that for any $g \in \Lambda$ we also have $-g \in \Lambda$, the Cayley multigraph on $G$ with connection multiset $\Lambda$, denoted by $\operatorname{Cay}[G: \Lambda]$, is the graph with vertex set $G$ and where the multiplicity of an edge $[x, y]$ is the multiplicity of $x-y$ in $\Lambda$. We point out that if $G=\mathbb{Z}_{v}$ then $\operatorname{Cay}\left[\mathbb{Z}_{v}: \Lambda\right]$ is also called a circulant multigraph.

In this paper we want to describe a general strategy to solve $\operatorname{BHR}(L)$ when the underlying-set of $L$ is $\{1,2, t\}$ for any arbitrary even integer $t \geqslant 4$.

All the known results about $\operatorname{BHR}(L)$-problem have been obtained thanks to cyclic and linear realizations. A cyclic realization of a list $L$ with $v-1$ elements each from the set $\left\{1, \ldots,\left\lfloor\frac{v}{2}\right\rfloor\right\}$ is a Hamiltonian path $P$ of $K_{v}$ such that the multiset of edge-lengths of $P$ equals $L$. Hence, it is clear that $\operatorname{BHR}(L)$ can be also formulated as: every such a list 
$L$ has a cyclic realization if and only if condition (1) is satisfied. For example, the path $[0,6,5,1,9,7,3,2,8,4]$ is a cyclic realization of $L=\left\{1^{2}, 2^{2}, 4^{5}\right\}$.

A linear realization of a list $L$ with $v-1$ positive integers not exceeding $v-1$ is a Hamiltonian path $\left[x_{0}, x_{1}, \ldots, x_{v-1}\right]$ of $K_{v}$ such that $L=\left\{\left|x_{i}-x_{i+1}\right| \mid i=0, \ldots, v-2\right\}$. For instance, one can easily check that the path $[0,8,7,6,4,2,10,9,1,3,5]$ is a linear realization of $L=\left\{1^{3}, 2^{4}, 8^{3}\right\}$.

We denote by $c L$ and $r L$ a cyclic and a linear realization of $L$, respectively. In this paper we shall choose 0 as first vertex of any path.

Remark 4. Every linear realization of a list $L$ can be viewed as a cyclic realization of a suitable list $L^{\prime}$, but not necessarily of the same list. Anyway if all the elements in the list are less than or equal to $\left\lfloor\frac{\lfloor L \mid+1}{2}\right\rfloor$, then every linear realization of $L$ is also a cyclic realization of the same list $L$ (see Section 3 of [6]).

In Section 2 we introduce two new classes of linear realizations and we show how they are fundamental to solve $\operatorname{BHR}(L)$-conjecture, in particular, when an element of $L$ is not a fixed number as in the case investigated in this paper. In Section 3 we construct linear realizations of $L=\left\{1,2^{b}, t^{c}\right\}$, while in Section 4 , using the results of previous sections, we construct linear realizations for the general case $L=\left\{1^{a}, 2^{b}, t^{c}\right\}$ with $a \geqslant 2$. Finally, in Section 5 we present a complete solution of $\operatorname{BHR}\left(\left\{1^{a}, 2^{b}, t^{c}\right\}\right)$, for $t=4,6,8$.

The main result of the paper is the following.

Theorem 5. If $t \geqslant 4$ is an even integer, then $\operatorname{BHR}\left(\left\{1^{a}, 2^{b}, t^{c}\right\}\right)$ holds for any $a, b, c \geqslant 1$ with $a+b \geqslant t-1$.

\section{Special linear realizations}

In this section we introduce two particular kinds of linear realizations which turned out to be very useful for solving $\operatorname{BHR}\left(\left\{1^{a}, 2^{b}, t^{c}\right\}\right)$.

Definition 6. Given a list $L$, we will say that a linear realization $r L$ is

- special of type 1 if $|L|$ and $|L|-1$ are adjacent in $r L$;

- special of type 2 if its endpoints are 0 and 1.

For short, we will denote by $S_{1} L$ and $S_{2} L$ a special linear realization of $L$ of type 1 and 2 , respectively. Also, by $S_{1,2} L$ we will mean a special linear realization of $L$ of both type 1 and 2 .

Now we will show how starting from a special linear realization of a given list $L$ it is possible to obtain a special linear realization of an infinite class of lists.

Lemma 7. If a list $L$ admits a special linear realization of type 1 , then also the list $L^{\prime}=L \cup\left\{2^{b}\right\}$ admits a special linear realization of type 1 , for any positive integer $b$. 
Proof. Set $v=|L|+1$ and let $S_{1} L=[0, \ldots, v-1, v-2, \ldots]$. Consider the realization obtained from $S_{1} L$ by adding the element $v$ between $v-1$ and $v-2$, namely $S_{1} L^{\prime}=$ $[0, \ldots, v-1, v, v-2, \ldots]$. It is easy to see that $S_{1} L^{\prime}$ is a special linear realization of type 1 of $L^{\prime}=L \cup\{2\}$. In order to obtain a $S_{1}\left(L \cup\left\{2^{b}\right\}\right)$ it is sufficient to apply the above process $b$ times. If $S_{1} L=[0, \ldots, v-2, v-1, \ldots]$ the thesis can be obtained in the same way.

Example 8. Consider $S_{1}\left\{1,2^{15}, 16\right\}=[0,2,4,6,8,10,12,14,16,17,1,3,5,7,9,11,13,15]$. Following the proof of Lemma 7 if we are looking, for instance, for $S_{1}\left\{1,2^{18}, 16\right\}$ we have to apply three times the process, obtaining $[0,2,4,6,8,10,12,14,16, \mathbf{1 8}, \mathbf{2 0}, \mathbf{1 9}, 17,1,3,5,7$, $9,11,13,15]$.

Lemma 9. If a list $L$ admits a special linear realization of type 2 , then also the list $L^{\prime}=L \cup\left\{2^{b}\right\}$ admits a special linear realization of type 2 , for any integer $b \geqslant 2$.

Proof. Let $S_{2} L=\left[0, x_{1}, x_{2}, \ldots, x_{n}, 1\right]$ and consider $S_{2} L^{\prime}=\left[0,2, x_{1}+2, x_{2}+2, \ldots, x_{n}\right.$ $+2,3,1]$, obtained from $S_{2} L$ by prepending 0 and appending 1 to the translate of $S_{2} L$ by 2. It is easy to see that $S_{2} L^{\prime}$ is a special linear realization of type 2 of $L^{\prime}=L \cup\left\{2^{2}\right\}$. So, if $b$ is even, in order to obtain a $S_{2}\left(L \cup\left\{2^{b}\right\}\right)$ it is sufficient to repeat the process $\frac{b}{2}$ times. Now consider $S_{2} L^{\prime \prime}=\left[0,2,4, x_{n}+3, \ldots, x_{2}+3, x_{1}+3,3,1\right]$, obtainable via the following steps: translate $S_{2} L$ by 3 , prepend 1 , append 2,0 and reverse. It is not hard to see that $S_{2} L^{\prime \prime}$ is a special linear realization of type 2 of $L^{\prime \prime}=L \cup\left\{2^{3}\right\}$. Hence, if $b$ is odd, say $b=2 k+3$, in order to obtain a $S_{2}\left(L \cup\left\{2^{b}\right\}\right)$ it is sufficient to apply $k$ times the first process and once the second process.

Example 10. Starting from $S_{2}\left\{1,2^{6}, 8^{2}\right\}=[0,8,6,4,2,3,5,7,9,1]$ we can obtain, for example, $S_{2}\left\{1,2^{8}, 8^{2}\right\}=[0,2,10,8,6,4,5,7,9,11,3,1]$ and $S_{2}\left\{1,2^{9}, 8^{2}\right\}=[0,2,4,12,10,8,6$, $5,7,9,11,3,1]$.

Lemma 11. If there exists a special linear realization of type 1 of a list $L_{1}$ and a special linear realization of type 2 of a list $L_{2}$, then there exists a linear realization of $L_{1} \cup L_{2}$.

Proof. Set $v=\left|L_{1}\right|+1$. Let $S_{1} L_{1}=\left[0, y_{1}, \ldots, y_{r}, v-2, v-1, z_{1}, \ldots, z_{s}\right]$ and $S_{2} L_{2}=$ $\left[0, x_{1}, x_{2}, \ldots, x_{n}, 1\right]$. Now consider $r L^{\prime}=\left[0, y_{1}, \ldots, y_{r}, v-2, x_{1}+v-2, x_{2}+v-2, \ldots, x_{n}+\right.$ $\left.v-2, v-1, z_{1}, \ldots, z_{s}\right]$. One can easily check that $r L^{\prime}$ is a linear realization of $L^{\prime}=L_{1} \cup L_{2}$. If $S_{1} L_{1}=\left[0, y_{1}, \ldots, y_{r}, v-1, v-2, z_{1}, \ldots, z_{s}\right]$ the thesis can be obtained in a similar way.

\section{Construction of linear realizations of $\left\{1,2^{b}, t^{c}\right\}$}

First of all we make some remarks which will be very useful in the following. Since the case in which the list has exactly two elements has been completely solved in [5] and [6], we consider through all the paper $\left\{1^{a}, 2^{b}, t^{c}\right\}$ with $t \geqslant 4$ an even integer and $a, b, c \geqslant 1$.

Remark 12. If there exists a linear realization $r L=\left[0, x_{1}, x_{2}, \ldots, x_{s}\right]$ of $L=\left\{1^{a}, 2^{b}, t^{c}\right\}$, then we have a linear realization of $L^{\prime}=\left\{1^{a+A}, 2^{b}, t^{c}\right\}$ for any $A \geqslant 0$. In fact, it suffices to consider $r L^{\prime}=\left[0,1,2, \ldots, A, x_{1}+A, x_{2}+A, \ldots, x_{s}+A\right]$. 
Lemma 13. If a list $L=\left\{1^{a_{1}}, 2^{a_{2}}, \ldots, t^{a_{t}}\right\}$ admits a linear realization $r L$, then $a_{i}+i-1 \leqslant$ $|L|$ for all $i=1, \ldots, t$.

Proof. By way of contradiction, suppose that there exists an integer $j$ such that $a_{j}+j-1>$ $|L|$. This means that $\sum_{i \neq j} a_{i}=|L|-a_{j}<j-1$. Remove from the path $r L$ the edges of length $i$ with $i \neq j$. In such a way we obtain at most $j-1$ connected components whose vertices belong to the same congruence class modulo $j$. Hence, we have a partition of the $v=|L|+1$ elements of $\mathbb{Z}_{v}$ in at most $j-1$ congruence classes modulo $j$, which is clearly an absurd.

Remark 14. In view of previous lemma, since we are looking for linear realizations of $L=\left\{1^{a}, 2^{b}, t^{c}\right\}$, in the following we will suppose $a+b \geqslant t-1$.

Notation. In order to present our realizations in a short way we will always use the notation here explained. In every realization of a list $L=\left\{1^{a}, 2^{b}, t^{c}\right\}$ the symbol " $x \stackrel{+i}{\rightarrow} y$ " means the arithmetic progression $x, x+i, x+2 i, \ldots, y-i, y$, where $x<y$ are congruent modulo $i$. Analogous meaning for the symbol " $x \stackrel{-i}{\rightarrow} y$ ", when $x>y$. If $i=t$, for short, by " $x \rightarrow y$ " we will mean the arithmetic progression $x, x+t, x+2 t, \ldots, y-t, y$ or the arithmetic progression $x, x-t, x-2 t, \ldots, y+t, y$ according to whether $y>x$ or $y<x$, respectively.

In view of Remark 12 it is natural to start investigating the case $a=1$, namely we construct linear realizations of $\left\{1,2^{b}, t^{c}\right\}$. In order to reduce the number of realizations we need to describe, we introduce a particular class of linear realizations and two related lemmas.

Definition 15. Given $L=\left\{1^{a}, 2^{b}, t^{c}\right\}$, set $v=|L|+1, e_{1}=[v-t, v-t+2]$ and $e_{2}=[v-t, v-t+1]$. A linear realization $r L$ is $t^{4 w}$-extendable if, for $i=1$ or $i=2$, both $e_{i}+4 x$ and $e_{i}+4 x+i$ are edges of $r L$ for any $x=0, \ldots, w$.

Lemma 16. If there exists a $t^{4 w}$-extendable linear realization $r L$ of $L=\left\{1^{a}, 2^{b}, t^{c}\right\}$, then there exists a linear realization of $L^{\prime}=\left\{1^{a}, 2^{b}, t^{c+4 x+4}\right\}$ for any $x=0, \ldots, w$. Furthermore, if $r L$ is special of type 2 , also $r L^{\prime}$ is special of type 2 .

Proof. If $e_{1}$ and $e_{1}+1$ are edges of $r L$, we replace $e_{1}$ with $[v-t, v, v+2, v-t+2]$ and $e_{1}+1$ with $[v-t+1, v+1, v+3, v-t+3]$. If $e_{2}$ and $e_{2}+2$ are edges of $r L$, we replace $e_{2}$ with $[v-t, v, v+1, v-t+1]$ and $e_{2}+2$ with $[v-t+2, v+2, v+3, v-t+3]$. In this way, we obtain a linear realization of $\left\{1^{a}, 2^{b}, t^{c+4}\right\}$. Now, we reapply this process to the edges $e_{1}+4$ and $e_{1}+5$ (or to $e_{2}+4$ and $e_{2}+6$, respectively), obtaining a linear realization of $\left\{1^{a}, 2^{b}, t^{c+8}\right\}$. Applying this process $x+1$ times (with $0 \leqslant x \leqslant w$ ) we obtain a linear realization of $\left\{1^{a}, 2^{b}, t^{c+4 x+4}\right\}$.

Example 17. It is easy to see that $S_{1} L=[0 \stackrel{+2}{\rightarrow} 14,15,1 \stackrel{+2}{\rightarrow} 13]$ is a $t^{8}$-extendable linear realization of $L=\left\{1,2^{13}, 14\right\}$ (consider the edges $[2,4]$ and $[3,5] ;[6,8]$ and $[7,9]$; $[10,12]$ and $[11,13])$. Applying once the construction illustrated in the proof of Lemma 
16, we obtain $r L^{\prime}=[0,2, \mathbf{1 6}, \mathbf{1 8}, 4 \stackrel{+2}{\longrightarrow} 14,15,1,3, \mathbf{1 7}, \mathbf{1 9}, 5 \stackrel{+2}{\longrightarrow} 13]$ which is a realization of $L^{\prime}=\left\{1,2^{13}, 14^{5}\right\}$. For example, if we are looking for a linear realization of $L^{\prime \prime}=\left\{1,2^{13}, 14^{9}\right\}$ we have to apply the process to $r L^{\prime}: r L^{\prime \prime}=[0,2,16,18,4,6, \mathbf{2 0}, \mathbf{2 2}, 8 \stackrel{+2}{\longrightarrow}$ $14,15,1,3,17,19,5,7, \mathbf{2 1}, \mathbf{2 3}, 9 \stackrel{+2}{\longrightarrow} 13]$. Finally, a linear realization of the list $L^{\prime \prime \prime}=$ $\left\{1,2^{13}, 14^{13}\right\}$ is $r L^{\prime \prime \prime}=[0,2,16,18,4,6,20,22,8,10, \mathbf{2 4}, \mathbf{2 6}, 12,14,15,1,3,17,19,5,7,21$, $23,9,11, \mathbf{2 5}, \mathbf{2 7}, 13]$.

Lemma 18. Let $t$ be even and suppose that $r L$ is a $t^{4 w}$-extendable linear realization of a list $L=\left\{1^{a}, 2^{b}, t^{c}\right\}$ for $w=\left\lfloor\frac{t}{4}\right\rfloor-1$. Then, we have the following:

(i) If $t \equiv 0(\bmod 4)$, then for any $k \geqslant 1$ there exists a $t^{4 w}$-extendable linear realization of $L^{\prime}=\left\{1^{a}, 2^{b}, t^{t k+c}\right\}$.

(ii) If $t \equiv 2(\bmod 4)$ and $r L$ is special of type 1 , then for any $k \geqslant 1$ there exists a $t^{4 w}$-extendable special linear realization of $L^{\prime}=\left\{1^{a}, 2^{b}, t^{t k+c}\right\}$ of type 1 .

(iii) If $r L$ is special of type 1 , then for any $y \geqslant 1$ there exists a $t^{4 w}$-extendable special realization of $L^{\prime}=\left\{1^{a}, 2^{b+4 y}, t^{c}\right\}$ of type 1 .

Proof. To prove (i) and (ii), clearly it suffices to consider the case $L^{\prime}=\left\{1^{a}, 2^{b}, t^{t+c}\right\}$. Let $t=4 u$ or $4 u+2$, so $w=u-1$. Since $r L$ is $t^{4 w}$-extendable, the edges $[v-t, v-t+2]$, $[v-$ $t+1, v-t+3], \ldots,[v-t+4 u-4, v-t+4 u-2],[v-t+4 u-3, v-t+4 u-1]$ are edges of $r L$, where $v=|L|+1$. Applying the process described in Lemma $16 w$ times, we obtain a linear realization of $L^{\prime \prime}=\left\{1^{a}, 2^{b}, t^{c+4 u}\right\}$, which contains the following subpaths: $[v-t, v, v+2, v-t+2],[v-t+1, v+1, v+3, v-t+3], \ldots,[v-t+4 u-4, v+4 u-$ $4, v+4 u-2, v-t+4 u-2],[v-t+4 u-3, v+4 u-3, v+4 u-1, v-t+4 u-1]$. Thus, $[v+4 x, v+2+4 x]$ and $[v+1+4 x, v+3+4 x]$ are edges of $r L^{\prime \prime}$ for any $x=0, \ldots, w$. Hence, $r L^{\prime \prime}$ is $t^{4 w}$-extendable.

If $t=4 u$, taking $L^{\prime}=L^{\prime \prime}$, we prove (i). If $t=4 u+2$, replace in $r L^{\prime \prime}$ either $[v-2, v-1]$ by $[v-2, v+t-2, v+t-1, v-1]$ or $[v-1, v-2]$ by $[v-1, v+t-1, v+t-2, v-2]$ (this is possible, since we are assuming that $r L$ is special of type 1). In this way we obtain a special linear realization of type 1 of $L^{\prime}=\left\{1^{a}, 2^{b}, t^{c+4 u+2}\right\}$, proving (ii).

To prove (iii), clearly it suffices to consider the case $L^{\prime}=\left\{1^{a}, 2^{b+4}, t^{c}\right\}$. Assume $S_{1} L=$ $\left[0, y_{1}, \ldots, y_{r}, v-2, v-1, z_{1}, \ldots, z_{s}\right]$, where again $v=|L|+1$, and let $S_{1} L^{\prime}$ be the realization obtained from $S_{1} L$ following the proof of Lemma 7 , namely $S_{1} L^{\prime}=\left[0, y_{1}, \ldots, y_{r}, v-\right.$ $\left.2, v, v+2, v+3, v+1, v-1, z_{1}, \ldots, z_{s}\right]$; clearly $\left|L^{\prime}\right|=v+3$. Since $S_{1} L$ is $t^{4 u-4}$-extendable, the edges $[v-t, v-t+2],[v-t+1, v-t+3], \ldots,[v-t+4(u-1), v-t+2+4(u-$ $1)],[v-t+1+4(u-1), v-t+3+4(u-1)]$ are edges of $S_{1} L$. So $S_{1} L^{\prime}$ contains the edges $[v+4-t, v+4-t+2],[v+4-t+1, v+4-t+3], \ldots,[v-t+4(u-1), v-t+2+$ $4(u-1)],[v-t+1+4(u-1), v-t+3+4(u-1)]$. If $t=4 u$, also the edges $[v, v+2]$ and $[v+1, v+3]$ appears in $S_{1} L^{\prime}$; while if $t=4 u+2$ the edges $[v-2, v]$ and $[v-1, v+1]$ are edges of $S_{1} L^{\prime}$. So, also $S_{1} L^{\prime}$ is $t^{4 u-4}$-extendable.

If the edges of $r L$ are $[v-t+4 x, v-t+1+4 x]$ and $[v-t+2+4 x, v-t+3+4 x]$, the statements can be proved in a similar way. 
Now we construct linear realizations of $L=\left\{1,2^{b}, t^{c}\right\}$. Writing $c=t k+d$ with $k \geqslant 0$ and $0 \leqslant d<t$, we distinguish two cases, according to the parity of $d$.

Proposition 19. Let $t \geqslant 4$ and $d \geqslant 0$ be even integers. The list $\left\{1,2^{b}, t^{t k+d}\right\}$ admits a special linear realization of type 2 for any $b \geqslant t-1$ if $d \equiv 2(\bmod 4)$ and $t \equiv 2(\bmod 4)$; for any $b \geqslant t-2$ otherwise.

Proof. Suppose firstly $t \equiv 0(\bmod 4)$ and consider the following special linear realizations of type 2 :

$$
\begin{array}{llrl}
S_{1,2}\left\{1,2^{t-2}\right\} & =[0 \stackrel{+2}{\rightarrow} t-2, t-1 \stackrel{-2}{\rightarrow} 1], & & S_{2}\left\{1,2^{t-2}, t^{2}\right\}=[0, t \stackrel{-2}{\rightarrow} 2,3 \stackrel{+2}{\rightarrow} t+1,1], \\
S_{1,2}\left\{1,2^{t-1}\right\} & =[0 \stackrel{+2}{\rightarrow} t, t-1 \stackrel{-2}{\longrightarrow} 1], & S_{2}\left\{1,2^{t-1}, t^{2}\right\} & =[0,2, t+2 \stackrel{-2}{\rightarrow} 4,3 \stackrel{+2}{\rightarrow} t+1,1] .
\end{array}
$$

It is not hard to check that all the above realizations are $t^{t-4}$-extendable. In fact in each case, the realization contains the edges $[v-t+4 x, v-t+2+4 x]$ and $[v-t+1+4 x, v-t+3+4 x]$ for any $x=0, \ldots, \frac{t-4}{4}$. Hence by Lemmas 16 and 18(i), the existence of the two first special linear realizations implies that of a special linear realization of type 2 of $\left\{1,2^{t-2}, t^{c}\right\}$ and of $\left\{1,2^{t-1}, t^{c}\right\}$, respectively, for any positive $c \equiv 0(\bmod 4)$. Also, the existence of the third and forth special linear realizations implies that of a special linear realization of type 2 of $\left\{1,2^{t-2}, t^{c}\right\}$ and of $\left\{1,2^{t-1}, t^{c}\right\}$, respectively, for any $c \equiv 2(\bmod 4)$.

Now from Lemma 9 we have that the existence of all these linear realizations ensures that of a special linear realization of type 2 of $\left\{1,2^{b}, t^{c}\right\}$ for any $b \geqslant t-2$ and any positive even integer $c$.

Suppose now $t \equiv 2(\bmod 4), t \geqslant 6$. We have the following special linear realizations of both type 1 and 2 :

$$
\begin{aligned}
S_{1,2}\left\{1,2^{t-2}\right\} & =[0 \stackrel{+2}{\rightarrow} t-2, t-1 \stackrel{-2}{\rightarrow} 1], & S_{1,2}\left\{1,2^{t-1}, t^{2}\right\} & =[0, t \stackrel{-2}{\rightarrow} 2, t+2, t+1 \stackrel{-2}{\rightarrow} 1], \\
S_{1,2}\left\{1,2^{t-1}\right\} & =[0 \stackrel{+2}{\longrightarrow} t, t-1 \stackrel{-2}{\longrightarrow} 1], & S_{1,2}\left\{1,2^{t}, t^{2}\right\} & =[0, t \stackrel{-2}{\rightarrow} 2, t+2, t+3 \stackrel{-2}{\rightarrow} 1] .
\end{aligned}
$$

The reader can check that the above realizations are $t^{t-6}$-extendable, since they contain the edges $[v-t+4 x, v-t+2+4 x]$ and $[v-t+1+4 x, v-t+3+4 x]$ for any $x=0, \ldots, \frac{t-6}{4}$. Hence by Lemmas 16 and 18(ii) we have the existence of a special linear realization of type 2 for the following lists: $\left\{1,2^{t-2}, t^{t k+d}\right\}$ for any $d \equiv 0(\bmod 4) ;\left\{1,2^{t-1}, t^{c}\right\}$ for any $c$ even; $\left\{1,2^{t}, t^{t k+d}\right\}$ for any $d \equiv 2(\bmod 4)$. Thus, by Lemma 9 there exists a special linear realization of type 2 of $\left\{1,2^{b}, t^{t k+d}\right\}$ for any $b \geqslant t-2$ if $d \equiv 0(\bmod 4)$ and for any $b \geqslant t-1$ if $d \equiv 2(\bmod 4)$.

Proposition 20. Let $t \equiv 0(\bmod 4), t \geqslant 4$. The list $\left\{1,2^{b}, t^{c}\right\}$ admits a linear realization for any $b \geqslant t-2$ and any positive odd integer $c$.

Proof. If $t=4$ we have the following special linear realizations of type 2 :

$$
\begin{aligned}
& S_{2}\left\{1,2^{2}, 4^{4 k+1}\right\}=[0 \rightarrow 4 k+4,4 k+2 \rightarrow 2,3 \rightarrow 4 k+3,4 k+1 \rightarrow 1], \\
& S_{2}\left\{1,2^{3}, 4^{4 k+1}\right\}=[0,2 \rightarrow 4 k+2,4 k+4 \rightarrow 4,3 \rightarrow 4 k+3,4 k+5 \rightarrow 1], \\
& S_{2}\left\{1,2^{2}, 4^{4 k+3}\right\}=[0 \rightarrow 4 k+4,4 k+6 \rightarrow 2,3 \rightarrow 4 k+3,4 k+5 \rightarrow 1], \\
& S_{2}\left\{1,2^{3}, 4^{4 k+3}\right\}=[0,2 \rightarrow 4 k+6,4 k+4 \rightarrow 4,3 \rightarrow 4 k+7,4 k+5 \rightarrow 1] .
\end{aligned}
$$

Hence by Lemma 9 we have a linear realization of $L=\left\{1,2^{b}, 4^{c}\right\}$ for any $b \geqslant 2$ and any odd positive integer $c$. 
So, we can assume $t \geqslant 8$. Write $c=t k+d$ with $k \geqslant 0$ and $0 \leqslant d<t$. We split the proof into two parts according to the congruence class of $d$ modulo 4 .

i) Let $d \equiv 1(\bmod 4)$.

For $b=t+1, t+2,2 t-1,2 t$ we have

$$
\begin{aligned}
S_{1}\left\{1,2^{t+1}, t\right\} & =[0 \stackrel{+2}{\rightarrow} t+2, t+3, t+1,1 \stackrel{+2}{\rightarrow} t-1], \\
S_{1}\left\{1,2^{t+2}, t\right\} & =[0 \stackrel{+2}{\rightarrow} t+4, t+3, t+1,1 \stackrel{+2}{\rightarrow} t-1], \\
S_{1}\left\{1,2^{2 t-1}, t\right\} & =[0 \stackrel{+2}{\longrightarrow} 2 t, 2 t+1 \stackrel{-2}{\rightarrow} t+1,1 \stackrel{+2}{\rightarrow} t-1], \\
S_{1}\left\{1,2^{2 t}, t\right\} & =[0 \stackrel{+2}{\longrightarrow} 2 t+2,2 t+1 \stackrel{-2}{\longrightarrow} t+1,1 \stackrel{+2}{\rightarrow} t-1] .
\end{aligned}
$$

Observe that these four special realizations are $t^{t-4}$-extendable and so we can apply Lemmas 16 and 18(i). Also, by Lemma 18(iii), we are left to consider the following cases. For $b=t-2, t-1, t$ we have

$$
\begin{aligned}
r\left\{1,2^{t-2}, t\right\} & =[0, t \stackrel{-2}{\rightarrow} 2,1 \stackrel{+2}{\rightarrow} t-1], \\
r\left\{1,2^{t-1}, t\right\} & =[0 \stackrel{+2}{\rightarrow} t, t-1, t+1,1 \stackrel{+2}{\rightarrow} t-3], \\
S_{1}\left\{1,2^{t}, t\right\} & =[0 \stackrel{+2}{\rightarrow} t+2, t+1,1 \stackrel{+2}{\rightarrow} t-1], \\
r\left\{1,2^{t}, t^{5}\right\} & =[0,2, t+2 \stackrel{-2}{\rightarrow} 6, t+6, t+4,4,3,1, t+1 \stackrel{-2}{\longrightarrow} 5, t+5, t+3] .
\end{aligned}
$$

For any $z$ such that $0 \leqslant z \leqslant \frac{t-12}{4}$ we take

$$
\begin{aligned}
r\left\{1,2^{t+4 z+3}, t^{5}\right\}= & {[0 \stackrel{+2}{\rightarrow} 4 z+4, t+4 z+4 \stackrel{-2}{\rightarrow} 4 z+8, t+4 z+8, t+4 z+6,} \\
& 4 z+6,4 z+7 \stackrel{-2}{\longrightarrow} 1, t+1 \stackrel{-2}{\rightarrow} 4 z+9, t+4 z+9 \stackrel{-2}{\rightarrow} t+3], \\
r\left\{1,2^{t+4 z+4}, t^{5}\right\}= & {[0 \stackrel{+2}{\longrightarrow} 4 z+6, t+4 z+6 \stackrel{-2}{\longrightarrow} 4 z+10, t+4 z+10, t+4 z+8,} \\
& 4 z+8,4 z+7 \stackrel{-2}{\longrightarrow} 1, t+1 \stackrel{-2}{\longrightarrow} 4 z+9, t+4 z+9 \stackrel{-2}{\longrightarrow} t+3] .
\end{aligned}
$$

Now, for $b=2 t-5,2 t-4$ we have

$$
\begin{aligned}
& r\left\{1,2^{2 t-5}, t^{5}\right\}=[0 \stackrel{+2}{\rightarrow} t-4,2 t-4 \stackrel{-2}{\neg} t, 2 t, 2 t-2, t-2, t-1 \stackrel{-2}{\rightarrow} 1 \rightarrow 2 t+1 \stackrel{-2}{\neg} t+3], \\
& r\left\{1,2^{2 t-4}, t^{5}\right\}=[0 \stackrel{+2}{\longrightarrow} t-2,2 t-2 \stackrel{-2}{\longrightarrow} t+2,2 t+2,2 t, t, t-1 \stackrel{-2}{\longrightarrow} 1 \rightarrow 2 t+1 \stackrel{-2}{\longrightarrow} t+3] .
\end{aligned}
$$

By an easy but long check one can see that all the previous realizations, except $S_{1}\left\{1,2^{t}, t\right\}$ are $t^{t-4}$-extendable. Hence, we obtain the existence of a linear realization of $\left\{1,2^{b}, t^{c}\right\}$ for any $b \geqslant t-2$ and any $c \equiv 1(\bmod 4)$.

ii) Let $d \equiv 3(\bmod 4)$.

For $b=t+3, t+4,2 t+1,2 t+2$ we have

$$
\begin{aligned}
S_{1}\left\{1,2^{t+3}, t^{3}\right\} & =[0,2, t+2 \stackrel{-2}{\rightarrow} 4, t+4, t+6, t+7 \stackrel{-2}{\rightarrow} t+1,1 \stackrel{+2}{\rightarrow} t-1], \\
S_{1}\left\{1,2^{t+4}, t^{3}\right\} & =[0,2,4, t+4 \stackrel{-2}{\rightarrow} 6, t+6, t+8, t+7 \stackrel{-2}{\rightarrow} t+1,1 \stackrel{+2}{\rightarrow} t-1], \\
S_{1}\left\{1,2^{2 t+1}, t^{3}\right\} & =[0 \stackrel{+2}{\rightarrow} 2 t+4,2 t+5 \stackrel{-2}{\rightarrow} t+7,7 \stackrel{+2}{\rightarrow} t+1,1,3,5, t+5, t+3], \\
S_{1}\left\{1,2^{2 t+2}, t^{3}\right\} & =[0 \stackrel{+2}{\longrightarrow} 2 t+6,2 t+5 \stackrel{-2}{\longrightarrow} t+7,7 \stackrel{+2}{\rightarrow} t+1,1,3,5, t+5, t+3] .
\end{aligned}
$$

Observe that these four special realizations are $t^{t-4}$-extendable and so we can apply Lemmas 16 and 18(i). Also, by Lemma 18(iii), we are left to consider the following cases. For $b=t-2, t-1, t, 2 t-3,2 t-2$ we have

$$
\begin{aligned}
r\left\{1,2^{t-2}, t^{3}\right\} & =[0, t, t+2,2 \stackrel{+2}{\rightarrow} t-2, t-1, t+1,1 \stackrel{+2}{\rightarrow} t-3], \\
r\left\{1,2^{t-1}, t^{3}\right\} & =[0, t, t+2,2 \stackrel{+2}{\rightarrow} t-2, t-1 \stackrel{-2}{\rightarrow} 1, t+1, t+3], \\
r\left\{1,2^{t}, t^{3}\right\} & =[0,2, t+2, t+4,4 \stackrel{+2}{\rightarrow} t, t-1 \stackrel{-2}{\rightarrow} 1, t+1, t+3], \\
r\left\{1,2^{2 t-3}, t^{3}\right\} & =[0 \stackrel{+2}{\rightarrow} t, 2 t \stackrel{-2}{\rightarrow} t+2, t+3 \stackrel{+2}{\rightarrow} 2 t+1 \rightarrow 1 \stackrel{+2}{\rightarrow} t-1], \\
r\left\{1,2^{2 t-2}, t^{3}\right\} & =[0 \stackrel{+2}{\rightarrow} t+2,2 t+2 \stackrel{-2}{\rightarrow} t+4, t+3 \stackrel{+2}{\rightarrow} 2 t+1 \rightarrow 1 \stackrel{+2}{\rightarrow} t-1] .
\end{aligned}
$$


For any $y$ with $0 \leqslant y \leqslant \frac{t-8}{4}$, we have the following linear realizations:

$$
\begin{aligned}
& r\left\{1,2^{t+4 y+1}, t^{3}\right\}=[0 \stackrel{+2}{\rightarrow} 4 y+4, t+4 y+4 \stackrel{-2}{\rightarrow} 4 y+6,4 y+7 \stackrel{+2}{\rightarrow} t+1,1 \stackrel{+2}{\rightarrow} 4 y+5, t+4 y+5 \stackrel{-2}{\rightarrow} t+3], \\
& r\left\{1,2^{t+4 y+2}, t^{3}\right\}=[0 \stackrel{+2}{\rightarrow} 4 y+6, t+4 y+6 \stackrel{-2}{\rightarrow} 4 y+8,4 y+7 \stackrel{+2}{\rightarrow} t+1,1 \stackrel{+2}{\rightarrow} 4 y+5, t+4 y+5 \stackrel{-2}{\rightarrow} t+3] .
\end{aligned}
$$

By an easy but long check one can see that the last seven realizations are $t^{t-4}$-extendable. So by Lemma 18(i) we obtain the existence of a linear realization of $\left\{1,2^{b}, t^{c}\right\}$ for any $b \geqslant t-2$ and any $c \equiv 3(\bmod 4)$.

Proposition 21. Let $t \equiv 2(\bmod 4), t \geqslant 6$ and let $c$ be an odd integer. Write $c=t k+d$, with $k \geqslant 0$ and $0 \leqslant d<t$. The list $\left\{1,2^{b}, t^{t k+d}\right\}$ admits a linear realization for any $b \geqslant t-1$ if $d \equiv 1(\bmod 4)$; for any $b \geqslant t-2$ if $d \equiv 3(\bmod 4)$.

Proof. First, consider the case $t=6$. We have the following linear realizations:

$$
\begin{aligned}
r\left\{1,2^{4}, 6^{6 k+1}\right\} & =[0 \rightarrow 6 k+6,6 k+4 \rightarrow 4,2 \rightarrow 6 k+2,6 k+1 \rightarrow 1,3 \rightarrow 6 k+3,6 k+5 \rightarrow 5], \\
S_{1}\left\{1,2^{4}, 6^{6 k+3}\right\} & =[0 \rightarrow 6 k+6,6 k+4 \rightarrow 4,2 \rightarrow 6 k+8,6 k+7 \rightarrow 1,3 \rightarrow 6 k+3,6 k+5 \rightarrow 5], \\
S_{1}\left\{1,2^{4}, 6^{6 k+5}\right\} & =[0 \rightarrow 6 k+6,6 k+8 \rightarrow 2,4 \rightarrow 6 k+10,6 k+9 \rightarrow 3,1 \rightarrow 6 k+7,6 k+5 \rightarrow 5], \\
S_{1}\left\{1,2^{5}, 6^{6 k+1}\right\} & =[0,2 \rightarrow 6 k+2,6 k+4 \rightarrow 4,6 \rightarrow 6 k+6,6 k+7 \rightarrow 1,3 \rightarrow 6 k+3,6 k+5 \rightarrow 5] .
\end{aligned}
$$

Hence by Lemma 7 we have the existence of a linear realization of $L=\left\{1,2^{b}, 6^{c}\right\}$ for any $b \geqslant 4$ and any odd integer $c$. So from now on, we can assume $t \geqslant 10$. We split the proof into two parts according to the congruence class of $d$ modulo 4 .

i) Let $d \equiv 1(\bmod 4)$.

For $b=t-1, t, t+5, t+6$ we have the following special realizations of type 1 :

$$
\begin{aligned}
S_{1}\left\{1,2^{t-1}, t\right\} & =[0 \stackrel{+2}{\rightarrow} t, t+1,1 \stackrel{+2}{\rightarrow} t-1], \\
S_{1}\left\{1,2^{t}, t\right\} & =[0 \stackrel{+2}{\rightarrow} t+2, t+1,1 \stackrel{+2}{\rightarrow} t-1], \\
S_{1}\left\{1,2^{t+5}, t^{5}\right\} & =[0 \stackrel{+2}{\rightarrow} 8, t+8 \stackrel{-2}{\longrightarrow} 10, t+10, t+11, t+9, t+7,7 \stackrel{+2}{\rightarrow} t+1,1,3,5, t+5, t+3], \\
S_{1}\left\{1,2^{t+6}, t^{5}\right\} & =[0 \stackrel{+2}{\longrightarrow} 10, t+10 \stackrel{-2}{\longrightarrow} 12, t+12, t+11, t+9, t+7,7 \stackrel{+2}{\longrightarrow} t+1,1,3,5, t+5, t+3] .
\end{aligned}
$$

Observe that the previous four special realizations of type 1 are $t^{t-6}$-extendable, so we can apply Lemmas 16 and 18(ii). Furthermore, by Lemma 7 we can assume $c>1$ and by Lemma 18(iii) we are left to consider only linear realizations for $b=t+1, t+2$ :

$$
\begin{aligned}
r\left\{1,2^{t+1}, t^{5}\right\}= & {[0,2,4, t+4, t+6,6 \stackrel{+2}{\rightarrow} t+2, t+3,3,1, t+1 \stackrel{-2}{\rightarrow} 5, t+5, t+7], } \\
r\left\{1,2^{t+2}, t^{5}\right\}= & {[0,2,4,6, t+6, t+8,8 \stackrel{+2}{\rightarrow} t+4, t+3,3,1, t+1 \stackrel{-2}{\rightarrow} 5, t+5, t+7], } \\
S_{1}\left\{1,2^{t+1}, t^{t+1}\right\}= & {[0,2,4 \rightarrow t+4, t+6 \rightarrow 6,8 \rightarrow t+8, \ldots, t-2 \rightarrow 2 t-2,2 t \rightarrow t,} \\
& t+2 \rightarrow 2 t+2,2 t+3 \rightarrow 3,1 \rightarrow 2 t+1,2 t-1 \rightarrow t-1, t-3 \rightarrow 2 t-3, \\
& 2 t-5 \rightarrow t-5, \ldots, t+9 \rightarrow 9,7,5 \rightarrow t+5, t+7], \\
S_{1}\left\{1,2^{t+2}, t^{t+1}\right\}= & {[0,2,4,6 \rightarrow t+6, t+8 \rightarrow 8,10 \rightarrow t+10, \ldots, t \rightarrow 2 t, 2 t+2 \rightarrow t+2,} \\
& t+4 \rightarrow 2 t+4,2 t+3 \rightarrow 3,1 \rightarrow 2 t+1,2 t-1 \rightarrow t-1, t-3 \rightarrow 2 t-3, \\
& 2 t-5 \rightarrow t-5, \ldots, t+9 \rightarrow 9,7,5 \rightarrow t+5, t+7] .
\end{aligned}
$$

The first two linear realizations are $t^{t-10}$-extendable, whereas the last two are special of type 1 and $t^{t-6}$-extendable. Hence, we proved the existence of a linear realization of $\left\{1,2^{b}, t^{t k+d}\right\}$ for any $b \geqslant t-1$ and $d \equiv 1(\bmod 4)$.

ii) Let $d \equiv 3(\bmod 4)$.

For $b=t-2, t+1, t+3, t+4$ we have the following special linear realizations of type 1 :

$$
\begin{aligned}
& S_{1}\left\{1,2^{t-2}, t^{3}\right\}=[0, t \stackrel{-2}{\longrightarrow} 2, t+2, t+1,1 \stackrel{+2}{\rightarrow} t-1], \\
& S_{1}\left\{1,2^{t+1}, t^{3}\right\}=[0,2, t+2 \stackrel{-2}{\rightarrow} 4, t+4, t+5, t+3, t+1,1 \stackrel{+2}{\rightarrow} t-1], \\
& S_{1}\left\{1,2^{t+3}, t^{3}\right\}=[0 \stackrel{+2}{\rightarrow} t+6, t+7,7 \stackrel{+2}{\rightarrow} t+1,1,3,5, t+5, t+3], \\
& S_{1}\left\{1,2^{t+4}, t^{3}\right\}=[0 \stackrel{+2}{\longrightarrow} t+8, t+7,7 \stackrel{+2}{\longrightarrow} t+1,1,3,5, t+5, t+3] .
\end{aligned}
$$


Observe that these four linear realizations are $t^{t-6}$-extendable. By Lemmas 16 and 18(iii), we are left to consider the following linear realizations for $b=t-1, t$ :

$$
\begin{aligned}
r\left\{1,2^{t-1}, t^{t k+3}\right\}= & {[0 \rightarrow t k+t, t k+t+2 \rightarrow 2,4 \rightarrow t k+4, t k+6 \rightarrow 6, \ldots, t-2 \rightarrow t k+t-2,} \\
& t k+t-1 \rightarrow t-1, t-3 \rightarrow t k+t-3, \ldots, t k+5 \rightarrow 5,3,1 \rightarrow t k+t+1, \\
& t k+t+3 \rightarrow t+3] \\
r\left\{1,2^{t}, t^{t k+3}\right\}= & {[0,2 \rightarrow t k+t+2, t k+t+4 \rightarrow 4,6 \rightarrow t k+6, t k+8 \rightarrow 8, \ldots, t \rightarrow t k+t,} \\
& t k+t-1 \rightarrow t-1, t-3 \rightarrow t k+t-3, \ldots, t k+5 \rightarrow 5,3,1 \rightarrow t k+t+1, \\
& t k+t+3 \rightarrow t+3] .
\end{aligned}
$$

Observe that the two previous realizations are $t^{t-10}$-extendable. Hence, by Lemma 16 we obtain a linear realization of $\left\{1,2^{b}, t^{t k+d}\right\}$ for any $b \geqslant t-2$ and any $d \equiv 3(\bmod 4)$.

Remark 22. Note that in previous propositions we have not constructed a linear realization of $\left\{1,2^{t-2}, t^{t k+d}\right\}$ when $t \equiv 2(\bmod 4)$ and $d \equiv 1,2(\bmod 4)$. However we point out that there exists a cyclic realization of both $\left\{1,2^{t-2}, t^{t k+t+4 x+1}\right\}$ and $\left\{1,2^{t-2}, t^{t k+t+4 x+2}\right\}$, with $0<4 x+1,4 x+2<t$. Namely, we have

$$
\begin{aligned}
r\left\{1,2^{t-2}, t^{t k+1}\right\}= & {[0 \rightarrow t k+t, t k+t-2 \rightarrow t-2, \ldots, t k+4 \rightarrow 4,2 \rightarrow t k+2,} \\
& t k+1 \rightarrow 1,3 \rightarrow t k+3, \ldots, t k+t-1 \rightarrow t-1], \\
c\left\{1^{1}, 2^{t-2}, t^{t k+t+4 x+5}\right\}= & {[0 \rightarrow t k+2 t, t k+2 t+2 \rightarrow 2,4 \rightarrow t k+2 t+4, \ldots, t k+2 t+4 x+2 \rightarrow} \\
& 4 x+2,4 x+4 \rightarrow t k+2 t+4 x+4,1 \rightarrow t k+2 t+1, t k+2 t+3 \rightarrow 3, \\
& \ldots, 4 x+1 \rightarrow t k+2 t+4 x+1, t k+2 t+4 x+3 \rightarrow 4 x+3,4 x+5 \rightarrow \\
& t k+t+4 x+5, t k+t+4 x+7 \rightarrow 4 x+7, \ldots, t-1 \rightarrow t k+t-1, \\
& t k+t-2 \rightarrow t-2, t-4 \rightarrow t k+t-4, \ldots, 4 x+6 \rightarrow t k+t+4 x+6], \\
S_{2}\left\{1,2^{t-2}, t^{t k+2}\right\}= & {[0 \rightarrow t k+t, t k+t-2 \rightarrow t-2, \ldots, t k+4 \rightarrow 4,2 \rightarrow t k+2,} \\
& t k+3 \rightarrow 3,5 \rightarrow t k+5, \ldots, t-1 \rightarrow t k+t-1, t k+t+1 \rightarrow 1], \\
c\left\{1,2^{t-2}, t^{t k+t+4 x+6}\right\}= & {[0,2 \rightarrow t k+2 t+2, t k+2 t+4 \rightarrow 4,6 \rightarrow t k+2 t+6, t k+2 t+8 \rightarrow 8,} \\
& \ldots, 4 x+2 \rightarrow t k+2 t+4 x+2, t k+2 t+4 x+4 \rightarrow 4 x+4,4 x+6 \rightarrow \\
& t k+t+4 x+6, t k+t+4 x+8 \rightarrow 4 x+8, \ldots, t \rightarrow t k+2 t, t k+2 t+1 \\
& \rightarrow 1,3 \rightarrow t k+2 t+3, t k+2 t+5 \rightarrow 5,7 \rightarrow t k+2 t+7, \ldots, 4 x+3 \rightarrow \\
& t k+2 t+4 x+3, t-3, t-5 \rightarrow t k+2 t-5, t k+2 t-7 \rightarrow t-7, \ldots, \\
& 4 x+5 \rightarrow t k+2 t+4 x+5, t-1 \rightarrow t k+2 t-1, t k+2 t-3 \rightarrow 2 t-3] .
\end{aligned}
$$

\section{Construction of linear realizations of $\left\{1^{a}, 2^{b}, t^{c}\right\}$ for $a \geqslant 2$}

In this section we investigate $\operatorname{BHR}\left(\left\{1^{a}, 2^{b}, t^{c}\right\}\right)$ when $a \geqslant 2$ and $a+b \geqslant t-1$. In view of Remark 12, we are left to consider only the cases when $a+b=t-1$.

Proposition 23. There exists a linear realization of $L=\left\{1^{a}, 2^{b}, t^{c}\right\}$ for any even integer $t \geqslant 4$ and any positive integer $a, b, c$ with $a \geqslant 2$ and $a+b=t-1$.

Proof. Since by hypothesis $a+b=t-1$ and, obviously, we have $t$ congruence classes modulo $t$, we use \pm 1 or \pm 2 to change the congruence class. As said above, all our linear realizations start from 0 , so adding $t$ a suitable number of times, we take all the elements of the congruence class 0 modulo $t$. Then applying \pm 1 or \pm 2 we go into another congruence class modulo $t$ and we take all the elements of this class adding or subtracting $t$. Next, we change again class applying \pm 1 or \pm 2 and so on. In this way we have to reach all the 
congruence classes modulo $t$, namely all the elements from 0 to $|L|$, using exactly $a$ times $1, b$ times 2 and $c$ times $t$.

In order to obtain a complete solution constructing a few number of realizations we look for those which are $t^{4 w}$-extendable. In particular, all the linear realizations of $\left\{1^{a}, 2^{b}, t^{c}\right\}$ we are going to construct with $a+b=t-1$ and $c=0,1,2,3$ are $t^{4 w}$-extendable with $w=\left\lfloor\frac{t}{4}\right\rfloor-1$. Then applying Lemmas 16 and 18 we have a linear realization for any list $\left\{1^{a}, 2^{b}, t^{c}\right\}$ with $a+b=t-1$ and $c \geqslant 1$.

Case 1. Let $a \equiv 0(\bmod 4)$.

We start with the case $t \equiv 0(\bmod 4)$, set $t=4 u \geqslant 8$. So we consider the list $L=$ $\left\{1^{4 y+4}, 2^{t-4 y-5}, t^{c}\right\}$ with $0 \leqslant y \leqslant u-2$ (since $\left.1 \leqslant a \leqslant t-2\right)$.

A linear realization of $L$ for $c=0$ and $c=2$ is, respectively

$$
P_{1}=[0 \stackrel{+1}{\rightarrow} 4 y+3 \stackrel{+2}{\longrightarrow} 4 u-1,4 u-2 \stackrel{-2}{\rightarrow} 4 y+4]
$$

and

$$
P_{2}=[0, t, t+1,1 \stackrel{+1}{\rightarrow} 4 y+1,4 y+3,4 y+2,4 y+4,4 y+5 \stackrel{+2}{\rightarrow} 4 u-1,4 u-2 \stackrel{-2}{\rightarrow} 4 y+6] .
$$

Furthermore, starting from the realization $P_{1}$ we can obtain a linear realization for $c=1$ by changing the sign of each integer $+1,-1,+2,-2$ used to change the congruence classes modulo $t$; observe that now $|L|=t$. Also, starting from the realization $P_{2}$ we obtain a linear realization for $c=3$, again by changing the sign of each integer $+1,-1,+2,-2$; note that now $|L|=t+2$.

Suppose now $t \equiv 2(\bmod 4)$; set $t=4 u-2 \geqslant 6$. Starting from the realization constructed above for $L$, when $t=4 u \geqslant 8$, we obtain a realization for $\left\{1^{4 y+4}, 2^{t-4 y-5}, t^{c}\right\}$ when $t=4 u-2$. If $c=0$, it is sufficient to replace the subpath $[4 u-3,4 u-1,4 u-2,4 u-4]$ of $P_{1}$ with the edge [4u-3,4u-4].

Consider $c=2$. If $y \leqslant u-3$, then replace in $P_{2}$ the subpath $[4 u-3,4 u-1,4 u-2,4 u-4]$ with the edge $[4 u-3,4 u-4]$.

If $y=u-2$, i.e. $L=\left\{1^{t-2}, 2, t^{2}\right\}$, we have the following linear realization:

$$
S_{1} L=[0, t, t+1,1 \stackrel{+1}{\rightarrow} t-3, t-1, t-2] .
$$

If $c=1$, the realization can be obtained as done for the case $t \equiv 0(\bmod 4)$, namely it is sufficient to change the signs of the all 1's and 2's used to construct the realization when $c=0$. Finally, if $c=3$ a linear realization is

$$
P_{3}=[0, t \stackrel{-1}{\rightarrow} t-4 y-3 \stackrel{-2}{\longrightarrow} 1, t+1, t+2,2 \stackrel{+2}{\rightarrow} t-4 y-4] .
$$

At this point we have obtained a linear realization for $\left\{1^{4 y+4}, 2^{t-4 y-5}, t^{c}\right\}$, for all even $t \geqslant 8$ and any positive integer $c$.

Case 2. Let $a \equiv 2(\bmod 4)$.

We start with the case $t \equiv 0(\bmod 4)$, set $t=4 u \geqslant 4$. So we consider the list $L=$ $\left\{1^{4 y+2}, 2^{t-4 y-3}, t^{c}\right\}$ with $0 \leqslant y \leqslant u-1$ (since $1 \leqslant a \leqslant t-2$ ). 
If $t \geqslant 8$ and $0 \leqslant y \leqslant u-2$, to obtain a linear realization of $L=\left\{1^{4 y+2}, 2^{t-4 y-3}\right\}$, replace in $P_{1}$ the subpath $[4 y \stackrel{+1}{\rightarrow} 4 y+3]$ with $[4 y, 4 y+2,4 y+1,4 y+3]$.

If $t \geqslant 4$ and $y=u-1$ (i.e. $L=\left\{1^{t-2}, 2\right\}$ ) we have

$$
S_{1} L=[0 \stackrel{+1}{\rightarrow} t-3, t-1, t-2] .
$$

Applying the reasoning explained in Case 1, we have also a linear realization of $\left\{1^{4 y+2}, 2^{t-4 y-3}, t\right\}$. Now, a linear realization of $L=\left\{1^{4 y+2}, 2^{t-4 y-3}, t^{2}\right\}$ is

$$
P_{4}=[0, t, t+1,1 \stackrel{+1}{\longrightarrow} 4 y+1 \stackrel{+2}{\longrightarrow} 4 u-1,4 u-2 \stackrel{-2}{\longrightarrow} 4 y+2]
$$

and from it we can also obtain a linear realization of $\left\{1^{4 y+2}, 2^{t-4 y-3}, t^{3}\right\}$, again changing the signs of 1's and 2's.

As in Case 1 , to solve the case $t=4 u-2 \geqslant 6$, we start from the realizations of $L$ when $t=4 u \geqslant 8$. In fact, in order to obtain a linear realization of $\left\{1^{4 y+2}, 2^{t-4 y-3}\right\}$ when $t=4 u-2 \geqslant 6$, it is sufficient to replace in $r\left\{1^{4 y+2}, 2^{t-4 y-3}\right\}$ constructed for $t=4 u$, the subpath $[4 u-3,4 u-1,4 u-2,4 u-4]$ with $[4 u-3,4 u-4]$.

Analogously, in order to obtain a linear realization of $\left\{1^{4 y+2}, 2^{t-4 y-3}, t^{2}\right\}$ when $t=$ $4 u-2 \geqslant 6$, it is sufficient to replace in $P_{4}$ the subpath $[4 u-3,4 u-1,4 u-2,4 u-4]$ with $[4 u-3,4 u-4]$.

As before, if $c=1$ a $r L$ can be obtained changing the signs of all the 1's and 2's used to obtain the realization in the case $c=0$. Finally, if $c=3$, we replace in $P_{3}$ the subpath $[t \stackrel{-1}{\rightarrow} t-3]$ with $[t, t-2, t-1, t-3]$.

Case 3. Let $a \equiv 3(\bmod 4)$.

We start with the case $t \equiv 0(\bmod 4)$, set $t=4 u \geqslant 8$. So we consider the list $L=$ $\left\{1^{4 y+3}, 2^{t-4 y-4}, t^{c}\right\}$ with $0 \leqslant y \leqslant u-2($ since $1 \leqslant a \leqslant t-2)$. A linear realization for $c=0$ and for $c=2$ is, respectively,

$$
P_{5}=[0 \stackrel{+1}{\longrightarrow} 4 y+1,4 y+3,4 y+2 \stackrel{+2}{\longrightarrow} 4 u-2,4 u-1 \stackrel{-2}{\rightarrow} 4 y+5]
$$

and

$$
P_{6}=[0, t, t+1,1 \stackrel{+1}{\rightarrow} 4 y+2 \stackrel{+2}{\rightarrow} 4 u-2,4 u-1 \stackrel{-2}{\rightarrow} 4 y+3] .
$$

The reader can check that, reasoning as in Case 1 , it is possible to construct a linear realization of $L$ for $c=1$ and $c=3$ starting from the realization $P_{5}$ and $P_{6}$, respectively.

Also here, to solve the case $t=4 u-2 \geqslant 6$, we start from the realizations of $L$ when $t=4 u \geqslant 8$. In fact, in order to obtain a linear realization of $\left\{1^{4 y+3}, 2^{t-4 y-4}\right\}$ when $t=4 u-2 \geqslant 6$, it is sufficient to replace in $P_{5}$ the subpath $[4 u-4,4 u-2,4 u-1,4 u-3]$ with $[4 u-4,4 u-3]$.

Analogously, in order to obtain a linear realization of $\left\{1^{4 y+3}, 2^{t-4 y-4}, t^{2}\right\}$ when $t=$ $4 u-2 \geqslant 6$, it is sufficient to replace in $P_{6}$ the subpath $[4 u-4,4 u-2,4 u-1,4 u-3]$ with $[4 u-4,4 u-3]$.

As before, if $c=1$ a $r L$ can be obtained changing the signs of all 1's and 2's used in the case $c=0$. Finally, if $c=3$, a linear realization of $L$ is

$$
P_{7}=[0, t, t-1, t-3, t-2, t-4 \stackrel{-1}{\longrightarrow} t-4 y-4 \stackrel{-2}{\rightarrow} 2, t+2, t+1,1 \stackrel{+2}{\rightarrow} t-4 y-5] .
$$


Case 4. Let $a \equiv 1(\bmod 4)$.

We start again with the case $t \equiv 0(\bmod 4)$, set $t=4 u \geqslant 8$. Since $a \geqslant 2$, we can assume $a=4 y+5$ with $0 \leqslant y \leqslant u-2$.

From the linear realization $P_{5}$ of $\left\{1^{4 y+3}, 2^{t-4 y-4}\right\}$ we can obtain a linear realization of $\left\{1^{4 y+5}, 2^{t-4 y-6}\right\}$. It suffices to replace in $P_{5}$ the subpath $[4 y+1,4 y+3,4 y+2,4 y+4]$ with $[4 y+1 \stackrel{+1}{\rightarrow} 4 y+4]$.

Moreover, if $c=2$, a linear realization of $\left\{1^{4 y+5}, 2^{t-4 y-6}, t^{2}\right\}$ is

$$
P_{8}=[0, t, t+1,1 \stackrel{+1}{\longrightarrow} 4 y+2,4 y+4,4 y+3,4 y+5,4 y+6 \stackrel{+2}{\longrightarrow} 4 u-2,4 u-1 \stackrel{-2}{\longrightarrow} 4 y+7] .
$$

Also in this case the reader can check that it is possible to obtain a linear realization of $\left\{1^{4 y+5}, 2^{t-4 y-6}, t^{c}\right\}$ for $c=1$ and $c=3$ changing the signs of the 1's and 2's used to construct the realization when $c=0$ and $c=2$, respectively.

Also here, to solve the case $t=4 u-2 \geqslant 10$, we start from the realizations of $L$ when $t=4 u \geqslant 12$. In fact, in order to obtain a linear realization of $\left\{1^{4 y+5}, 2^{t-4 y-6}\right\}$ when $t=4 u-2 \geqslant 10$, it suffices to replace in $r\left\{1^{4 y+5}, 2^{t-4 y-6}\right\}$ constructed for $t=4 u$ the subpath $[4 u-4,4 u-2,4 u-1,4 u-3]$ with $[4 u-4,4 u-3]$.

Analogously, in order to obtain a linear realization of $\left\{1^{4 y+5}, 2^{t-4 y-6}, t^{2}\right\}$ when $t=$ $4 u-2 \geqslant 10$, it suffices to replace in $P_{8}$ the subpath $[4 u-4,4 u-2,4 u-1,4 u-3]$ with $[4 u-4,4 u-3]$.

As before, if $c=1$ a $r L$ can be obtained changing the signs of all 1's and 2's used in the case $c=0$. Finally, if $c=3$, it suffices to replace in $P_{7}$ the subpath $[t-1, t-3, t-2, t-4]$ with $[t-1 \stackrel{-1}{\rightarrow} t-4]$.

Now we can prove the main result of this paper, that is Theorem 5 .

Proof of Theorem 5. We prove the theorem using the linear realizations constructed in Sections 3 and 4. Observe that since $t$ appears in $L=\left\{1^{a}, 2^{b}, t^{c}\right\}$, we have $|L| \geqslant 2 t-1$ and so we can apply Remark 4.

Let $t \equiv 0(\bmod 4)$. Combining Propositions 19 and 20 with Remark 12, we obtain a linear realization for every list $L$ with $a, c \geqslant 1$ and $b \geqslant t-2$. If $1 \leqslant b \leqslant t-3$, then $a \geqslant 2$ and hence the thesis follows from Proposition 23 and Remark 12 .

Now, let $t \equiv 2(\bmod 4)$. Write $c=t k+d$ with $k \geqslant 0$ and $0 \leqslant d<t$. Combining Propositions 19 and 21 with Remark 12, we obtain a linear realization for every list $\left\{1^{a}, 2^{b}, t^{t k+d}\right\}$ with $a \geqslant 1, b \geqslant t-2, k \geqslant 0$ and $d \equiv 0,3(\bmod 4)$.

Assume $d \equiv 1,2(\bmod 4)$. If $b \geqslant t-1$ proceed as before. When $a=1$ and $b=t-2$ we have a cyclic realization, see Remark 22. If $a=2$, we have the following $t^{t-6}$-extendable linear realizations:

$$
\begin{aligned}
S_{1}\left\{1^{2}, 2^{t-2}, t\right\} & =[0, t, t+1 \stackrel{-2}{\longrightarrow} 1,2 \stackrel{+2}{\longrightarrow} t-2], \\
S_{1}\left\{1^{2}, 2^{t-2}, t^{2}\right\} & =[0,2, t+2, t+1,1 \stackrel{+2}{\longrightarrow} t-1, t \stackrel{-2}{\longrightarrow} 4] .
\end{aligned}
$$

Hence by Lemmas 16 and 18(ii) we have a linear realization of $\left\{1^{2}, 2^{t-2}, t^{t k+d}\right\}$ and applying Remark 12 we have a linear realization of $\left\{1^{a}, 2^{t-2}, t^{t k+d}\right\}$ for any $a \geqslant 2$. Also in this case, if $1 \leqslant b \leqslant t-3$, then $a \geqslant 2$ and hence the thesis follows from Proposition 23 and Remark 12 . 


\section{A complete solution for $t=4,6,8$}

In view of Theorem 5 , in order to prove $\operatorname{BHR}\left(\left\{1^{a}, 2^{b}, t^{c}\right\}\right)$ for all $a, b, c \geqslant 1$ and $t$ even, we are left to construct cyclic realizations only when $a+b \leqslant t-2$. As examples, we prove $\operatorname{BHR}\left(\left\{1^{a}, 2^{b}, 4^{c}\right\}\right), \operatorname{BHR}\left(\left\{1^{a}, 2^{b}, 6^{c}\right\}\right)$ and $\operatorname{BHR}\left(\left\{1^{a}, 2^{b}, 8^{c}\right\}\right)$.

Proposition 24. $\operatorname{BHR}\left(\left\{1^{a}, 2^{b}, 4^{c}\right\}\right)$ holds for all $a, b, c \geqslant 1$.

Proof. Firstly, observe that since 4 appears as edge-length, then $v=a+b+c+1 \geqslant$ 8. Furthermore, the assumptions $a, b, c \geqslant 1$ reduce the conjecture to prove that $L=$ $\left\{1^{a}, 2^{b}, 4^{c}\right\}$ admits a cyclic realization except when 4 divides $v$ and $a+b=2$. If $a+b \geqslant 3$, Remark 4 and Theorem 5 imply the existence of such a realization of $L$. For $a+b=2$ (i.e., $a=b=1$ ) we have

$$
\begin{aligned}
& c\left\{1,2,4^{4 k+6}\right\}=[0 \rightarrow 4 k+8,3 \rightarrow 4 k+7,4 k+5 \rightarrow 1,2 \rightarrow 4 k+6], \\
& c\left\{1,2,4^{4 k+7}\right\}=[0 \rightarrow 4 k+8,2 \rightarrow 4 k+6,4 k+7 \rightarrow 3,1 \rightarrow 4 k+9], \\
& c\left\{1,2,4^{4 k+8}\right\}=[0 \rightarrow 4 k+8,1 \rightarrow 4 k+9,4 k+7 \rightarrow 3,2 \rightarrow 4 k+10] .
\end{aligned}
$$

Proposition 25. $\operatorname{BHR}\left(\left\{1^{a}, 2^{b}, 6^{c}\right\}\right)$ holds for all $a, b, c \geqslant 1$.

Proof. Firstly, observe that since 6 appears as edge-length, then $v=a+b+c+1 \geqslant$ 12. Furthermore, the assumptions $a, b, c \geqslant 1$ reduce the conjecture to prove that $L=$ $\left\{1^{a}, 2^{b}, 6^{c}\right\}$ admits a cyclic realization except when 6 divides $v$ and $a+b \leqslant 4$. If $a+b \geqslant 5$, Remark 4 and Theorem 5 imply the existence of such a realization of $L$. For $a+b \leqslant 4$ we have

$$
\begin{aligned}
c\left\{1,2,6^{6 k+10}\right\} & =[0 \rightarrow 6 k+12,5 \rightarrow 6 k+11,4 \rightarrow 6 k+10,3 \rightarrow 6 k+9,6 k+7 \rightarrow 1,2 \rightarrow 6 k+8], \\
c\left\{1,2,6^{6 k+11}\right\} & =[0 \rightarrow 6 k+12,4 \rightarrow 6 k+10,2 \rightarrow 6 k+8,6 k+9 \rightarrow 3,1 \rightarrow 6 k+13,5 \rightarrow 6 k+11], \\
c\left\{1,2,6^{6 k+12}\right\} & =[0 \rightarrow 6 k+12,3 \rightarrow 6 k+9,6 k+10 \rightarrow 4,6 k+13 \rightarrow 1,6 k+14 \rightarrow 2,6 k+11 \rightarrow 5], \\
c\left\{1,2,6^{6 k+13}\right\} & =[0 \rightarrow 6 k+12,2 \rightarrow 6 k+14,4 \rightarrow 6 k+10,6 k+11 \rightarrow 5,6 k+15 \rightarrow 3,1 \rightarrow 6 k+13], \\
c\left\{1,2,6^{6 k+14}\right\} & =[0 \rightarrow 6 k+12,1 \rightarrow 6 k+13,6 k+11 \rightarrow 5,6 k+16 \rightarrow 4,6 k+15 \rightarrow 3,2 \rightarrow 6 k+14], \\
c\left\{1,2^{2}, 6^{6 k+9}\right\} & =[0 \rightarrow 6 k+12,5 \rightarrow 6 k+11,4 \rightarrow 6 k+10,6 k+8 \rightarrow 2,1 \rightarrow 6 k+7,6 k+9 \rightarrow 3], \\
c\left\{1,2^{2}, 6^{6 k+10}\right\} & =[0 \rightarrow 6 k+12,4 \rightarrow 6 k+10,2 \rightarrow 6 k+8,6 k+9 \rightarrow 3,1 \rightarrow 6 k+13,6 k+11 \rightarrow 5], \\
c\left\{1,2^{2}, 6^{6 k+11}\right\} & =[0 \rightarrow 6 k+12,3 \rightarrow 6 k+9,6 k+10 \rightarrow 4,2 \rightarrow 6 k+14,5 \rightarrow 6 k+11,6 k+13 \rightarrow 1], \\
c\left\{1,2^{2}, 6^{6 k+12}\right\} & =[0 \rightarrow 6 k+12,2 \rightarrow 6 k+14,4 \rightarrow 6 k+10,6 k+11 \rightarrow 5,3 \rightarrow 6 k+15,1 \rightarrow 6 k+13], \\
c\left\{1,2^{2}, 6^{6 k+13}\right\} & =[0 \rightarrow 6 k+12,1 \rightarrow 6 k+13,6 k+11 \rightarrow 5,6 k+16 \rightarrow 4,2 \rightarrow 6 k+14,6 k+15 \rightarrow 3], \\
c\left\{1,2^{3}, 6^{6 k+8}\right\} & =[0 \rightarrow 6 k+12,5 \rightarrow 6 k+11,6 k+9 \rightarrow 3,1 \rightarrow 6 k+7,6 k+8 \rightarrow 2,4 \rightarrow 6 k+10], \\
c\left\{1,2^{3}, 6^{6 k+9}\right\} & =[0 \rightarrow 6 k+12,4 \rightarrow 6 k+10,6 k+8 \rightarrow 2,1 \rightarrow 6 k+13,6 k+11 \rightarrow 5,3 \rightarrow 6 k+9], \\
c\left\{1,2^{3}, 6^{6 k+10}\right\} & =[0 \rightarrow 6 k+12,3 \rightarrow 6 k+9,6 k+10 \rightarrow 4,2 \rightarrow 6 k+14,1 \rightarrow 6 k+13,6 k+11 \rightarrow 5], \\
c\left\{1,2^{3}, 6^{6 k+11}\right\} & =[0 \rightarrow 6 k+12,2 \rightarrow 6 k+14,4 \rightarrow 6 k+10,6 k+11 \rightarrow 5,7 \rightarrow 6 k+13,6 k+15 \rightarrow 3,1], \\
c\left\{1,2^{3}, 6^{6 k+12}\right\} & =[0 \rightarrow 6 k+12,1 \rightarrow 6 k+13,6 k+11 \rightarrow 5,3 \rightarrow 6 k+15,6 k+14 \rightarrow 2,4 \rightarrow 6 k+16], \\
c\left\{1^{2}, 2,6^{6 k+9}\right\} & =[0 \rightarrow 6 k+12,5 \rightarrow 6 k+11,4 \rightarrow 6 k+10,6 k+9 \rightarrow 3,1 \rightarrow 6 k+7,6 k+8 \rightarrow 2], \\
c\left\{1^{2}, 2,6^{6 k+10}\right\} & =[0 \rightarrow 6 k+12,4 \rightarrow 6 k+10,6 k+11 \rightarrow 5,6 k+13 \rightarrow 1,3 \rightarrow 6 k+9,6 k+8 \rightarrow 2], \\
c\left\{1^{2}, 2,6^{6 k+11}\right\} & =[0 \rightarrow 6 k+12,3 \rightarrow 6 k+9,6 k+11 \rightarrow 5,4 \rightarrow 6 k+10,1 \rightarrow 6 k+13,16 k+14 \rightarrow 2], \\
c\left\{1^{2}, 2,6^{6 k+12}\right\} & =[0 \rightarrow 6 k+12,2 \rightarrow 6 k+14,6 k+13 \rightarrow 1,3 \rightarrow 6 k+15,5 \rightarrow 6 k+11,6 k+10 \rightarrow 4], \\
c\left\{1^{2}, 2,6^{6 k+13}\right\} & =[0 \rightarrow 6 k+12,1 \rightarrow 6 k+13,6 k+11 \rightarrow 5,4 \rightarrow 6 k+16,6 k+15 \rightarrow 3,6 k+14 \rightarrow 2], \\
c\left\{1^{2}, 2^{2}, 6^{6 k+8}\right\} & =[0 \rightarrow 6 k+12,5 \rightarrow 6 k+11,6 k+9 \rightarrow 3,4 \rightarrow 6 k+10,6 k+8 \rightarrow 2,1 \rightarrow 6 k+7], \\
c\left\{1^{2}, 2^{2}, 6^{6 k+9}\right\} & =[0 \rightarrow 6 k+12,4 \rightarrow 6 k+10,6 k+9 \rightarrow 3,5 \rightarrow 6 k+11,6 k+13 \rightarrow 1,2 \rightarrow 6 k+8], \\
c\left\{1^{2}, 2^{2}, 6^{6 k+10}\right\} & =[0 \rightarrow 6 k+12,3 \rightarrow 6 k+9,6 k+10 \rightarrow 4,5 \rightarrow 6 k+11,6 k+13 \rightarrow 1,6 k+14 \rightarrow 2],
\end{aligned}
$$




$$
\begin{aligned}
c\left\{1^{2}, 2^{2}, 6^{6 k+11}\right\} & =[0 \rightarrow 6 k+12,2 \rightarrow 6 k+14,6 k+15 \rightarrow 3,1 \rightarrow 6 k+13,6 k+11 \rightarrow 5,4 \rightarrow 6 k+10], \\
c\left\{1^{2}, 2^{2}, 6^{6 k+12}\right\} & =[0 \rightarrow 6 k+12,1 \rightarrow 6 k+13,6 k+14 \rightarrow 2,4 \rightarrow 6 k+16,6 k+15 \rightarrow 3,5 \rightarrow 6 k+11], \\
c\left\{1^{3}, 2,6^{6 k+8}\right\} & =[0 \rightarrow 6 k+12,5 \rightarrow 6 k+11,6 k+10 \rightarrow 4,3 \rightarrow 6 k+9,6 k+7 \rightarrow 1,2 \rightarrow 6 k+8], \\
c\left\{1^{3}, 2,6^{6 k+9}\right\} & =[0 \rightarrow 6 k+12,4 \rightarrow 6 k+10,6 k+11 \rightarrow 5,3 \rightarrow 6 k+9,6 k+8 \rightarrow 2,1 \rightarrow 6 k+13], \\
c\left\{1^{3}, 2,6^{6 k+10}\right\} & =[0 \rightarrow 6 k+12,3 \rightarrow 6 k+9,6 k+10 \rightarrow 4,5 \rightarrow 6 k+11,6 k+13 \rightarrow 1,2 \rightarrow 6 k+14], \\
c\left\{1^{3}, 2,6^{6 k+11}\right\} & =[0 \rightarrow 6 k+12,2 \rightarrow 6 k+14,6 k+13 \rightarrow 1,6 k+15 \rightarrow 3,4 \rightarrow 6 k+10,6 k+11 \rightarrow 5], \\
c\left\{1^{3}, 2,6^{6 k+12}\right\} & =[0 \rightarrow 6 k+12,1 \rightarrow 6 k+13,6 k+11 \rightarrow 5,4 \rightarrow 6 k+16,6 k+15 \rightarrow 3,2 \rightarrow 6 k+14] .
\end{aligned}
$$

Proposition 26. $\operatorname{BHR}\left(\left\{1^{a}, 2^{b}, 8^{c}\right\}\right)$ holds for all $a, b, c \geqslant 1$.

Proof. Firstly, observe that since 8 appears as edge-length, then $v=a+b+c+1 \geqslant$ 16. Furthermore, the assumptions $a, b, c \geqslant 1$ reduce the conjecture to prove that $L=$ $\left\{1^{a}, 2^{b}, 8^{c}\right\}$ admits a cyclic realization except when either 4 divides $v$ and $a=b=1$ or 8 divides $v$ and $a+b \leqslant 6$. If $a+b \geqslant 7$, Remark 4 and Theorem 5 imply the existence of such a realization of $L$. For $a+b \leqslant 6$ we have

$$
\begin{aligned}
& c\left\{1,2,8^{8 k+14}\right\}=[0 \rightarrow 8 k+16,7 \rightarrow 8 k+15,6 \rightarrow 8 k+14,5 \rightarrow 8 k+13,4 \rightarrow 8 k+12,3 \rightarrow 8 k+11, \\
& 8 k+9 \rightarrow 1,2 \rightarrow 8 k+10] \\
& c\left\{1,2,8^{8 k+15}\right\}=[0 \rightarrow 8 k+16,6 \rightarrow 8 k+14,4 \rightarrow 8 k+12,2 \rightarrow 8 k+10,8 k+11 \rightarrow 3,1 \rightarrow 8 k+17, \\
& 7 \rightarrow 8 k+15,5 \rightarrow 8 k+13] \\
& c\left\{1,2,8^{8 k+16}\right\}=[0 \rightarrow 8 k+16,5 \rightarrow 8 k+13,2 \rightarrow 8 k+18,1 \rightarrow 8 k+17,6 \rightarrow 8 k+14,3 \rightarrow 8 k+11, \\
& 8 k+12 \rightarrow 4,8 k+15 \rightarrow 7] \\
& c\left\{1,2,8^{8 k+18}\right\}=[0 \rightarrow 8 k+16,3 \rightarrow 8 k+19,6 \rightarrow 8 k+14,1 \rightarrow 8 k+17,4 \rightarrow 8 k+12,8 k+13 \rightarrow 5, \\
& 8 k+18,8 k+20,7 \rightarrow 8 k+15,2 \rightarrow 8 k+10] \text {, } \\
& c\left\{1,2,8^{8 k+19}\right\}=[0 \rightarrow 8 k+16,2 \rightarrow 8 k+18,4 \rightarrow 8 k+20,6 \rightarrow 8 k+14,8 k+15 \rightarrow 7,8 k+21 \rightarrow 5, \\
& 8 k+19 \rightarrow 3,1 \rightarrow 8 k+17] \\
& c\left\{1,2,8^{8 k+20}\right\}=[0 \rightarrow 8 k+16,1 \rightarrow 8 k+17,2 \rightarrow 8 k+18,3 \rightarrow 8 k+19,4 \rightarrow 8 k+20,5 \rightarrow 8 k+5, \\
& 8 k+6 \rightarrow 6,8 k+21,8 k+13,8 k+15 \rightarrow 7,8 k+22,8 k+14] \text {, } \\
& c\left\{1,2^{2}, 8^{8 k+13}\right\}=[0 \rightarrow 8 k+16,7 \rightarrow 8 k+15,6 \rightarrow 8 k+14,5 \rightarrow 8 k+13,4 \rightarrow 8 k+12,8 k+10 \rightarrow \\
& 2,3 \rightarrow 8 k+11,8 k+9 \rightarrow 1] \\
& c\left\{1,2^{2}, 8^{8 k+14}\right\}=[0 \rightarrow 8 k+16,6 \rightarrow 8 k+14,4 \rightarrow 8 k+12,8 k+10 \rightarrow 2,1 \rightarrow 8 k+17,7 \rightarrow 8 k+15, \\
& 5 \rightarrow 8 k+13,8 k+11 \rightarrow 3] \text {, } \\
& c\left\{1,2^{2}, 8^{8 k+15}\right\}=[0 \rightarrow 8 k+16,5 \rightarrow 8 k+13,8 k+15 \rightarrow 7,8 k+18 \rightarrow 2,4 \rightarrow 8 k+12,8 k+11 \rightarrow 3, \\
& 8 k+14 \rightarrow 6,8 k+17 \rightarrow 1] \text {, } \\
& c\left\{1,2^{2}, 8^{8 k+16}\right\}=[0 \rightarrow 8 k+16,4 \rightarrow 8 k+12,8 k+14 \rightarrow 6,8 k+18 \rightarrow 2,3 \rightarrow 8 k+19,7 \rightarrow \\
& 8 k+15,8 k+13 \rightarrow 5,8 k+17 \rightarrow 1], \\
& c\left\{1,2^{2}, 8^{8 k+17}\right\}=[0 \rightarrow 8 k+16,3 \rightarrow 8 k+19,6 \rightarrow 8 k+14,8 k+13 \rightarrow 5,7 \rightarrow 8 k+15,2 \rightarrow \\
& 8 k+18,8 k+20 \rightarrow 4,8 k+17 \rightarrow 1] \text {, } \\
& c\left\{1,2^{2}, 8^{8 k+18}\right\}=[0 \rightarrow 8 k+16,2 \rightarrow 8 k+18,4 \rightarrow 8 k+20,6 \rightarrow 8 k+14,8 k+13 \rightarrow 5,7 \rightarrow 8 k+15, \\
& 1 \rightarrow 8 k+17,3 \rightarrow 8 k+19,8 k+21] \\
& c\left\{1,2^{2}, 8^{8 k+19}\right\}=[0 \rightarrow 8 k+16,1 \rightarrow 8 k+17,2 \rightarrow 8 k+18,3 \rightarrow 8 k+19,4 \rightarrow 8 k+20,8 k+21, \\
& 6 \rightarrow 8 k+22,7,5 \rightarrow 8 k+13,8 k+15 \rightarrow 15] \text {, } \\
& c\left\{1,2^{3}, 8^{8 k+12}\right\}=[0 \rightarrow 8 k+16,7 \rightarrow 8 k+15,6 \rightarrow 8 k+14,5 \rightarrow 8 k+13,8 k+11 \rightarrow 3,1 \rightarrow 8 k+9, \\
& 8 k+10 \rightarrow 2,4 \rightarrow 8 k+12] \text {, }
\end{aligned}
$$




$$
\begin{aligned}
& c\left\{1,2^{3}, 8^{8 k+13}\right\}=[0 \rightarrow 8 k+16,6 \rightarrow 8 k+14,4 \rightarrow 8 k+12,2 \rightarrow 8 k+10,8 k+11 \rightarrow 3,1 \rightarrow 8 k+17, \\
& 8 k+15 \rightarrow 7,5 \rightarrow 8 k+13] \\
& c\left\{1,2^{3}, 8^{8 k+14}\right\}=[0 \rightarrow 8 k+16,5 \rightarrow 8 k+13,2 \rightarrow 8 k+18,7 \rightarrow 8 k+15,8 k+17 \rightarrow 1,3 \rightarrow 8 k+11, \\
& 8 k+12 \rightarrow 4,6 \rightarrow 8 k+14], \\
& c\left\{1,2^{3}, 8^{8 k+15}\right\}=[0 \rightarrow 8 k+16,4 \rightarrow 8 k+12,8 k+14 \rightarrow 6,8 k+18 \rightarrow 2,1 \rightarrow 8 k+17,8 k+19 \rightarrow 3, \\
& 8 k+15 \rightarrow 7,5 \rightarrow 8 k+13] \\
& c\left\{1,2^{3}, 8^{8 k+16}\right\}=[0 \rightarrow 8 k+16,3 \rightarrow 8 k+19,6 \rightarrow 8 k+14,1 \rightarrow 8 k+17,8 k+18 \rightarrow 2,4 \rightarrow 8 k+20, \\
& 7,5 \rightarrow 8 k+13,8 k+15 \rightarrow 15] \text {, } \\
& c\left\{1,2^{3}, 8^{8 k+17}\right\}=[0 \rightarrow 8 k+16,2 \rightarrow 8 k+18,4 \rightarrow 8 k+20,6 \rightarrow 8 k+14,8 k+13 \rightarrow 5,8 k+19, \\
& 8 k+21,7 \rightarrow 8 k+15,8 k+17 \rightarrow 1,3 \rightarrow 8 k+11], \\
& c\left\{1,2^{3}, 8^{8 k+18}\right\}=[0 \rightarrow 8 k+16,1 \rightarrow 8 k+17,2 \rightarrow 8 k+18,3 \rightarrow 8 k+19,4 \rightarrow 8 k+20,8 k+22,7, \\
& 5,13,15 \rightarrow 8 k+15,8 k+14 \rightarrow 6,8 k+21 \rightarrow 21], \\
& c\left\{1,2^{4}, 8^{8 k+11}\right\}=[0 \rightarrow 8 k+16,7 \rightarrow 8 k+15,6 \rightarrow 8 k+14,8 k+12 \rightarrow 4,2 \rightarrow 8 k+10,8 k+9 \rightarrow 1, \\
& 3 \rightarrow 8 k+11,8 k+13 \rightarrow 5] \\
& c\left\{1,2^{4}, 8^{8 k+12}\right\}=[0 \rightarrow 8 k+16,6 \rightarrow 8 k+14,4 \rightarrow 8 k+12,8 k+10 \rightarrow 2,1 \rightarrow 8 k+17,8 k+15 \rightarrow 7, \\
& 5 \rightarrow 8 k+13,8 k+11 \rightarrow 3] \\
& c\left\{1,2^{4}, 8^{8 k+13}\right\}=[0 \rightarrow 8 k+16,5 \rightarrow 8 k+13,2 \rightarrow 8 k+18,7 \rightarrow 8 k+15,8 k+17 \rightarrow 1,3 \rightarrow 8 k+11, \\
& 8 k+12,8 k+14 \rightarrow 6,4 \rightarrow 8 k+4] \\
& c\left\{1,2^{4}, 8^{8 k+14}\right\}=[0 \rightarrow 8 k+16,4 \rightarrow 8 k+12,8 k+14 \rightarrow 6,8 k+18 \rightarrow 2,1 \rightarrow 8 k+17,8 k+19, \\
& 7 \rightarrow 8 k+15,8 k+13 \rightarrow 5,3 \rightarrow 8 k+11] \\
& c\left\{1,2^{4}, 8^{8 k+15}\right\}=[0 \rightarrow 8 k+16,3 \rightarrow 8 k+19,6 \rightarrow 8 k+14,1 \rightarrow 8 k+17,8 k+15 \rightarrow 7,5 \rightarrow 8 k+13, \\
& 8 k+12 \rightarrow 4,2 \rightarrow 8 k+18,8 k+20], \\
& c\left\{1,2^{4}, 8^{8 k+16}\right\}=[0 \rightarrow 8 k+16,2 \rightarrow 8 k+18,4 \rightarrow 8 k+20,6 \rightarrow 8 k+14,8 k+13 \rightarrow 5,7 \rightarrow 8 k+15, \\
& 1 \rightarrow 8 k+9,8 k+11 \rightarrow 3,8 k+17,8 k+19,8 k+21], \\
& c\left\{1,2^{4}, 8^{8 k+17}\right\}=[0 \rightarrow 8 k+16,1 \rightarrow 8 k+17,2 \rightarrow 8 k+18,8 k+20 \rightarrow 4,3 \rightarrow 8 k+19,8 k+21, \\
& 6 \rightarrow 8 k+22,7,5 \rightarrow 8 k+13,8 k+15 \rightarrow 15], \\
& c\left\{1,2^{5}, 8^{8 k+10}\right\}=[0 \rightarrow 8 k+16,7 \rightarrow 8 k+15,6 \rightarrow 8 k+14,8 k+12 \rightarrow 4,2 \rightarrow 8 k+10,8 k+9 \rightarrow 1, \\
& 3,5 \rightarrow 8 k+13,8 k+11 \rightarrow 11] \\
& c\left\{1,2^{5}, 8^{8 k+11}\right\}=[0 \rightarrow 8 k+16,6 \rightarrow 8 k+14,4 \rightarrow 8 k+12,2 \rightarrow 8 k+10,8 k+9 \rightarrow 1,3 \rightarrow 8 k+11, \\
& 8 k+13,8 k+15,8 k+17,7 \rightarrow 8 k+7,8 k+5 \rightarrow 5], \\
& c\left\{1,2^{5}, 8^{8 k+12}\right\}=[0 \rightarrow 8 k+16,5 \rightarrow 8 k+13,2 \rightarrow 8 k+10,8 k+12 \rightarrow 4,6 \rightarrow 8 k+14,3 \rightarrow 8 k+11, \\
& 8 k+9 \rightarrow 1,8 k+18,8 k+17,8 k+15 \rightarrow 7], \\
& c\left\{1,2^{5}, 8^{8 k+13}\right\}=[0 \rightarrow 8 k+16,4 \rightarrow 8 k+12,8 k+14 \rightarrow 6,8 k+18 \rightarrow 2,3 \rightarrow 8 k+11,8 k+9 \rightarrow 1, \\
& 8 k+13 \rightarrow 5,7 \rightarrow 8 k+15,8 k+17,8 k+19] \text {, } \\
& c\left\{1,2^{5}, 8^{8 k+14}\right\}=[0 \rightarrow 8 k+16,3 \rightarrow 8 k+19,6 \rightarrow 8 k+14,1 \rightarrow 8 k+17,4,2 \rightarrow 8 k+10, \\
& 8 k+12 \rightarrow 12,13 \rightarrow 8 k+13,8 k+15 \rightarrow 7,5,8 k+18,8 k+20], \\
& c\left\{1,2^{5}, 8^{8 k+15}\right\}=[0 \rightarrow 8 k+16,2 \rightarrow 8 k+18,4 \rightarrow 8 k+20,6 \rightarrow 8 k+14,8 k+15 \rightarrow 7,9 \rightarrow 8 k+17, \\
& 8 k+19,5 \rightarrow 8 k+13,8 k+11 \rightarrow 3,1,8 k+21] \text {, } \\
& c\left\{1,2^{5}, 8^{8 k+16}\right\}=[0 \rightarrow 8 k+16,1 \rightarrow 8 k+17,2 \rightarrow 8 k+18,8 k+20 \rightarrow 4,6 \rightarrow 8 k+6,8 k+5 \rightarrow 5, \\
& 3 \rightarrow 8 k+19,8 k+21,8 k+13,8 k+15 \rightarrow 7,8 k+22,8 k+14], \\
& c\left\{1^{2}, 2,8^{8 k+13}\right\}=[0 \rightarrow 8 k+16,7 \rightarrow 8 k+15,6 \rightarrow 8 k+14,5 \rightarrow 8 k+13,4 \rightarrow 8 k+12,3 \rightarrow 8 k+11, \\
& 8 k+9,8 k+10 \rightarrow 2,1 \rightarrow 8 k+1], \\
& c\left\{1^{2}, 2,8^{8 k+14}\right\}=[0 \rightarrow 8 k+16,6 \rightarrow 8 k+14,4 \rightarrow 8 k+12,8 k+13 \rightarrow 5,8 k+15 \rightarrow 7,8 k+17 \rightarrow 1, \\
& 3 \rightarrow 8 k+11,8 k+10 \rightarrow 2], \\
& c\left\{1^{2}, 2,8^{8 k+15}\right\}=[0 \rightarrow 8 k+16,5 \rightarrow 8 k+13,8 k+15 \rightarrow 7,8 k+18 \rightarrow 2,1 \rightarrow 8 k+17,6 \rightarrow 8 k+14, \\
& 3 \rightarrow 8 k+11,8 k+12 \rightarrow 4], \\
& c\left\{1^{2}, 2,8^{8 k+16}\right\}=[0 \rightarrow 8 k+16,4 \rightarrow 8 k+12,8 k+13 \rightarrow 5,8 k+17 \rightarrow 1,3 \rightarrow 8 k+19,7 \rightarrow 8 k+15, \\
& 8 k+14 \rightarrow 6,8 k+18 \rightarrow 2],
\end{aligned}
$$




$$
\begin{aligned}
& c\left\{1^{2}, 2,8^{8 k+17}\right\}=[0 \rightarrow 8 k+16,3 \rightarrow 8 k+19,6 \rightarrow 8 k+14,1 \rightarrow 8 k+17,8 k+18 \rightarrow 2,8 k+15, \\
& 8 k+13 \rightarrow 5,4 \rightarrow 8 k+20,7 \rightarrow 8 k+7], \\
& c\left\{1^{2}, 2,8^{8 k+18}\right\}=[0 \rightarrow 8 k+16,2 \rightarrow 8 k+18,8 k+19 \rightarrow 3,8 k+17 \rightarrow 1,8 k+15 \rightarrow 7,8 k+21 \rightarrow 5, \\
& 4 \rightarrow 8 k+12,8 k+14 \rightarrow 6,8 k+20] \text {, } \\
& c\left\{1^{2}, 2,8^{8 k+19}\right\}=[0 \rightarrow 8 k+16,1 \rightarrow 8 k+17,2 \rightarrow 8 k+18,3 \rightarrow 8 k+19,4 \rightarrow 8 k+20,8 k+22, \\
& 7 \rightarrow 8 k+15,8 k+14 \rightarrow 6,5 \rightarrow 8 k+21], \\
& c\left\{1^{2}, 2^{2}, 8^{8 k+12}\right\}=[0 \rightarrow 8 k+16,7 \rightarrow 8 k+15,6 \rightarrow 8 k+14,5 \rightarrow 8 k+13,8 k+12 \rightarrow 4,2 \rightarrow 8 k+10, \\
& 8 k+11 \rightarrow 3,1 \rightarrow 8 k+9] \\
& c\left\{1^{2}, 2^{2}, 8^{8 k+13}\right\}=[0 \rightarrow 8 k+16,6 \rightarrow 8 k+14,4 \rightarrow 8 k+12,8 k+13 \rightarrow 5,8 k+15 \rightarrow 7,8 k+17, \\
& 1 \rightarrow 8 k+9,8 k+11 \rightarrow 3,2 \rightarrow 8 k+10] \\
& c\left\{1^{2}, 2^{2}, 8^{8 k+14}\right\}=[0 \rightarrow 8 k+16,5 \rightarrow 8 k+13,8 k+15 \rightarrow 7,8 k+18 \rightarrow 2,1 \rightarrow 8 k+17,6 \rightarrow 8 k+14, \\
& 8 k+12 \rightarrow 4,3 \rightarrow 8 k+11] \\
& c\left\{1^{2}, 2^{2}, 8^{8 k+15}\right\}=[0 \rightarrow 8 k+16,4 \rightarrow 8 k+12,8 k+14 \rightarrow 6,5 \rightarrow 8 k+13,1 \rightarrow 8 k+17,8 k+15 \rightarrow 7, \\
& 8 k+19 \rightarrow 3,2 \rightarrow 8 k+18] \\
& c\left\{1^{2}, 2^{2}, 8^{8 k+16}\right\}=[0 \rightarrow 8 k+16,3 \rightarrow 8 k+19,6 \rightarrow 8 k+14,1 \rightarrow 8 k+17,8 k+18 \rightarrow 10,12 \rightarrow 8 k+20, \\
& 7 \rightarrow 8 k+15,2,4,5 \rightarrow 8 k+13] \\
& c\left\{1^{2}, 2^{2}, 8^{8 k+17}\right\}=[0 \rightarrow 8 k+16,2 \rightarrow 8 k+18,4 \rightarrow 8 k+20,8 k+19 \rightarrow 3,8 k+17 \rightarrow 1,8 k+21, \\
& 7 \rightarrow 8 k+15,8 k+13 \rightarrow 5,6 \rightarrow 8 k+14], \\
& c\left\{1^{2}, 2^{2}, 8^{8 k+18}\right\}=[0 \rightarrow 8 k+16,1 \rightarrow 8 k+17,2 \rightarrow 8 k+18,8 k+20 \rightarrow 4,8 k+19 \rightarrow 3,5,6, \\
& 8 k+21 \rightarrow 13,14 \rightarrow 8 k+22,7 \rightarrow 8 k+15] \\
& c\left\{1^{2}, 2^{3}, 8^{8 k+11}\right\}=[0 \rightarrow 8 k+16,7 \rightarrow 8 k+15,6 \rightarrow 8 k+14,5 \rightarrow 8 k+13,8 k+12 \rightarrow 4,2 \rightarrow 8 k+10, \\
& 8 k+11,8 k+9 \rightarrow 1,3 \rightarrow 8 k+3] \\
& c\left\{1^{2}, 2^{3}, 8^{8 k+12}\right\}=[0 \rightarrow 8 k+16,6 \rightarrow 8 k+14,4 \rightarrow 8 k+12,8 k+13 \rightarrow 5,8 k+15 \rightarrow 7,9 \rightarrow 8 k+17, \\
& 1,3 \rightarrow 8 k+11,8 k+10 \rightarrow 2], \\
& c\left\{1^{2}, 2^{3}, 8^{8 k+13}\right\}=[0 \rightarrow 8 k+16,5 \rightarrow 8 k+13,8 k+15 \rightarrow 7,8 k+18 \rightarrow 2,1 \rightarrow 8 k+9,8 k+11 \rightarrow 3, \\
& 4 \rightarrow 8 k+12,8 k+14 \rightarrow 6,8 k+17] \\
& c\left\{1^{2}, 2^{3}, 8^{8 k+14}\right\}=[0 \rightarrow 8 k+16,4 \rightarrow 8 k+12,8 k+14 \rightarrow 6,5 \rightarrow 8 k+13,8 k+15 \rightarrow 7,8 k+19 \rightarrow 3, \\
& 1 \rightarrow 8 k+17,8 k+18 \rightarrow 2] \\
& c\left\{1^{2}, 2^{3}, 8^{8 k+15}\right\}=[0 \rightarrow 8 k+16,3 \rightarrow 8 k+19,8 k+17 \rightarrow 1,2 \rightarrow 8 k+18,5 \rightarrow 8 k+13,8 k+15, \\
& 8 k+14 \rightarrow 6,4 \rightarrow 8 k+20,7 \rightarrow 8 k+7], \\
& c\left\{1^{2}, 2^{3}, 8^{8 k+16}\right\}=[0 \rightarrow 8 k+16,2 \rightarrow 8 k+18,4 \rightarrow 8 k+20,8 k+19 \rightarrow 3,5 \rightarrow 8 k+21, \\
& 1 \rightarrow 8 k+17,8 k+15 \rightarrow 7,6 \rightarrow 8 k+14], \\
& c\left\{1^{2}, 2^{3}, 8^{8 k+17}\right\}=[0 \rightarrow 8 k+16,1 \rightarrow 8 k+17,8 k+15 \rightarrow 7,5 \rightarrow 8 k+21,8 k+22 \rightarrow 6,4 \rightarrow 8 k+20, \\
& 8 k+19 \rightarrow 3,8 k+18 \rightarrow 2], \\
& c\left\{1^{2}, 2^{4}, 8^{8 k+10}\right\}=[0 \rightarrow 8 k+16,7 \rightarrow 8 k+15,6 \rightarrow 8 k+14,5 \rightarrow 8 k+13,8 k+11 \rightarrow 3,1,2, \\
& 4 \rightarrow 8 k+12,8 k+10 \rightarrow 10,9 \rightarrow 8 k+9], \\
& c\left\{1^{2}, 2^{4}, 8^{8 k+11}\right\}=[0 \rightarrow 8 k+16,6 \rightarrow 8 k+14,4 \rightarrow 8 k+12,8 k+13 \rightarrow 5,7 \rightarrow 8 k+15,8 k+17, \\
& 1 \rightarrow 8 k+9,8 k+11 \rightarrow 3,2 \rightarrow 8 k+10] \text {, } \\
& c\left\{1^{2}, 2^{4}, 8^{8 k+12}\right\}=[0 \rightarrow 8 k+16,5 \rightarrow 8 k+13,8 k+15 \rightarrow 7,8 k+18 \rightarrow 2,1,3,8 k+14 \rightarrow 6, \\
& 4 \rightarrow 8 k+12,8 k+11 \rightarrow 11,9 \rightarrow 8 k+17] \\
& c\left\{1^{2}, 2^{4}, 8^{8 k+13}\right\}=[0 \rightarrow 8 k+16,4 \rightarrow 8 k+12,8 k+13 \rightarrow 5,7 \rightarrow 8 k+15,3 \rightarrow 8 k+11, \\
& 8 k+9 \rightarrow 1,8 k+19,8 k+17,8 k+18,6 \rightarrow 8 k+14,2 \rightarrow 8 k+10], \\
& c\left\{1^{2}, 2^{4}, 8^{8 k+14}\right\}=[0 \rightarrow 8 k+16,3 \rightarrow 8 k+19,8 k+17 \rightarrow 1,2 \rightarrow 8 k+18,8 k+20 \rightarrow 4, \\
& 6 \rightarrow 8 k+14,8 k+15 \rightarrow 7,5 \rightarrow 8 k+13], \\
& c\left\{1^{2}, 2^{4}, 8^{8 k+15}\right\}=[0 \rightarrow 8 k+16,2 \rightarrow 8 k+18,8 k+20 \rightarrow 4,5 \rightarrow 8 k+21,8 k+19 \rightarrow 11 \text {, } \\
& 9 \rightarrow 8 k+17,3,1,8 k+15 \rightarrow 7,6 \rightarrow 8 k+14], \\
& c\left\{1^{2}, 2^{4}, 8^{8 k+16}\right\}=[0 \rightarrow 8 k+16,1 \rightarrow 8 k+17,8 k+15 \rightarrow 7,5 \rightarrow 8 k+21,8 k+22 \rightarrow 6, \\
& 4 \rightarrow 8 k+20,8 k+18 \rightarrow 2,3 \rightarrow 8 k+19] \text {, }
\end{aligned}
$$




$$
\begin{aligned}
& c\left\{1^{3}, 2,8^{8 k+12}\right\}=[0 \rightarrow 8 k+16,7 \rightarrow 8 k+15,6 \rightarrow 8 k+14,5 \rightarrow 8 k+13,8 k+12 \rightarrow 4, \\
& 3 \rightarrow 8 k+11,8 k+9 \rightarrow 1,2 \rightarrow 8 k+10], \\
& c\left\{1^{3}, 2,8^{8 k+13}\right\}=[0 \rightarrow 8 k+16,6 \rightarrow 8 k+14,4 \rightarrow 8 k+12,8 k+13 \rightarrow 5,8 k+15 \rightarrow 7,8 k+17, \\
& 1 \rightarrow 8 k+9,8 k+10 \rightarrow 2,3 \rightarrow 8 k+11] \\
& c\left\{1^{3}, 2,8^{8 k+14}\right\}=[0 \rightarrow 8 k+16,5 \rightarrow 8 k+13,8 k+15 \rightarrow 7,8 k+18 \rightarrow 2,1 \rightarrow 8 k+17, \\
& 6 \rightarrow 8 k+14,3,4 \rightarrow 8 k+12,8 k+11 \rightarrow 11] \\
& c\left\{1^{3}, 2,8^{8 k+15}\right\}=[0 \rightarrow 8 k+16,4 \rightarrow 8 k+12,8 k+14 \rightarrow 6,5 \rightarrow 8 k+13,1 \rightarrow 8 k+17, \\
& 8 k+18 \rightarrow 2,3 \rightarrow 8 k+19,7 \rightarrow 8 k+15], \\
& c\left\{1^{3}, 2,8^{8 k+16}\right\}=[0 \rightarrow 8 k+16,3 \rightarrow 8 k+19,8 k+18 \rightarrow 2,1 \rightarrow 8 k+17,4 \rightarrow 8 k+20, \\
& 7 \rightarrow 8 k+15,8 k+13 \rightarrow 5,6 \rightarrow 8 k+14], \\
& c\left\{1^{3}, 2,8^{8 k+17}\right\}=[0 \rightarrow 8 k+16,2 \rightarrow 8 k+18,4 \rightarrow 8 k+20,8 k+19 \rightarrow 3,8 k+17 \rightarrow 1, \\
& 8 k+21 \rightarrow 5,6 \rightarrow 8 k+14,8 k+15 \rightarrow 7], \\
& c\left\{1^{3}, 2,8^{8 k+18}\right\}=[0 \rightarrow 8 k+16,1 \rightarrow 8 k+17,2 \rightarrow 8 k+18,3 \rightarrow 8 k+19,4 \rightarrow 8 k+20,8 k+21 \text {, } \\
& 8 k+22 \rightarrow 6,7 \rightarrow 8 k+15,8 k+13 \rightarrow 5] \text {, } \\
& c\left\{1^{3}, 2^{2}, 8^{8 k+11}\right\}=[0 \rightarrow 8 k+16,7 \rightarrow 8 k+15,6 \rightarrow 8 k+14,8 k+13 \rightarrow 5,4 \rightarrow 8 k+12, \\
& 8 k+10 \rightarrow 2,3 \rightarrow 8 k+11,8 k+9 \rightarrow 1], \\
& c\left\{1^{3}, 2^{2}, 8^{8 k+12}\right\}=[0 \rightarrow 8 k+16,6 \rightarrow 8 k+14,4 \rightarrow 8 k+12,8 k+13 \rightarrow 5,3 \rightarrow 8 k+11, \\
& 8 k+10 \rightarrow 2,1 \rightarrow 8 k+17,8 k+15 \rightarrow 7], \\
& c\left\{1^{3}, 2^{2}, 8^{8 k+13}\right\}=[0 \rightarrow 8 k+16,5 \rightarrow 8 k+13,8 k+15 \rightarrow 7,8 k+18 \rightarrow 2,1 \rightarrow 8 k+17,6 \rightarrow 8 k+14, \\
& 8 k+12,8 k+11 \rightarrow 3,4 \rightarrow 8 k+4] \text {, } \\
& c\left\{1^{3}, 2^{2}, 8^{8 k+14}\right\}=[0 \rightarrow 8 k+16,4 \rightarrow 8 k+12,8 k+14 \rightarrow 6,5 \rightarrow 8 k+13,1 \rightarrow 8 k+17,8 k+15 \rightarrow 7, \\
& 8 k+19,8 k+18 \rightarrow 2,3 \rightarrow 8 k+11] \\
& c\left\{1^{3}, 2^{2}, 8^{8 k+15}\right\}=[0 \rightarrow 8 k+16,3 \rightarrow 8 k+19,8 k+17 \rightarrow 1,2 \rightarrow 8 k+18,5 \rightarrow 8 k+13,8 k+12 \rightarrow 4 \text {, } \\
& 6 \rightarrow 8 k+14,8 k+15 \rightarrow 7,8 k+20] \text {, } \\
& c\left\{1^{3}, 2^{2}, 8^{8 k+16}\right\}=[0 \rightarrow 8 k+16,2 \rightarrow 8 k+18,4 \rightarrow 8 k+20,8 k+19 \rightarrow 3,8 k+17 \rightarrow 1,8 k+21 \rightarrow 5,7, \\
& 6 \rightarrow 8 k+14,8 k+15 \rightarrow 15] \\
& c\left\{1^{3}, 2^{2}, 8^{8 k+17}\right\}=[0 \rightarrow 8 k+16,1 \rightarrow 8 k+17,2 \rightarrow 8 k+18,8 k+20 \rightarrow 4,8 k+19 \rightarrow 3,5 \rightarrow 8 k+13, \\
& 8 k+14,8 k+15 \rightarrow 7,8 k+22,8 k+21,6 \rightarrow 8 k+6] \\
& c\left\{1^{3}, 2^{3}, 8^{8 k+10}\right\}=[0 \rightarrow 8 k+16,7 \rightarrow 8 k+15,6 \rightarrow 8 k+14,8 k+13 \rightarrow 5,4 \rightarrow 8 k+12,8 k+10 \rightarrow 2, \\
& 3,1 \rightarrow 8 k+9,8 k+11 \rightarrow 11] \\
& c\left\{1^{3}, 2^{3}, 8^{8 k+11}\right\}=[0 \rightarrow 8 k+16,6 \rightarrow 8 k+14,4 \rightarrow 8 k+12,8 k+13 \rightarrow 5,3 \rightarrow 8 k+11,1,2 \rightarrow 8 k+10, \\
& 8 k+9 \rightarrow 9,7 \rightarrow 8 k+15,8 k+17] \\
& c\left\{1^{3}, 2^{3}, 8^{8 k+12}\right\}=[0 \rightarrow 8 k+16,5 \rightarrow 8 k+13,8 k+15 \rightarrow 7,8 k+18 \rightarrow 2,1 \rightarrow 8 k+17,6 \rightarrow 8 k+6, \\
& 8 k+4 \rightarrow 4,3 \rightarrow 8 k+11,8 k+12,8 k+14] \text {, } \\
& c\left\{1^{3}, 2^{3}, 8^{8 k+13}\right\}=[0 \rightarrow 8 k+16,4 \rightarrow 8 k+12,8 k+14 \rightarrow 6,5 \rightarrow 8 k+13,8 k+15 \rightarrow 7,8 k+19, \\
& 1 \rightarrow 8 k+17,8 k+18 \rightarrow 2,3 \rightarrow 8 k+11] \\
& c\left\{1^{3}, 2^{3}, 8^{8 k+14}\right\}=[0 \rightarrow 8 k+16,3 \rightarrow 8 k+19,8 k+17 \rightarrow 1,2 \rightarrow 8 k+18,8 k+20 \rightarrow 4, \\
& 5 \rightarrow 8 k+13,8 k+15 \rightarrow 7,6 \rightarrow 8 k+14], \\
& c\left\{1^{3}, 2^{3}, 8^{8 k+15}\right\}=[0 \rightarrow 8 k+16,2 \rightarrow 8 k+18,4 \rightarrow 8 k+20,8 k+19 \rightarrow 3,5 \rightarrow 8 k+13,8 k+14 \rightarrow 6, \\
& 7 \rightarrow 8 k+15,8 k+17 \rightarrow 1,8 k+21] \text {, } \\
& c\left\{1^{3}, 2^{3}, 8^{8 k+16}\right\}=[0 \rightarrow 8 k+16,1 \rightarrow 8 k+17,2 \rightarrow 8 k+18,8 k+20 \rightarrow 4,3 \rightarrow 8 k+19,8 k+21, \\
& 8 k+22,7 \rightarrow 8 k+15,8 k+13 \rightarrow 5,6 \rightarrow 8 k+14], \\
& c\left\{1^{4}, 2,8^{8 k+11}\right\}=[0 \rightarrow 8 k+16,7 \rightarrow 8 k+15,6 \rightarrow 8 k+14,5 \rightarrow 8 k+13,8 k+12 \rightarrow 4, \\
& 3 \rightarrow 8 k+11,8 k+9,8 k+10 \rightarrow 2,1 \rightarrow 8 k+1] \text {, } \\
& c\left\{1^{4}, 2,8^{8 k+12}\right\}=[0 \rightarrow 8 k+16,6 \rightarrow 8 k+14,4 \rightarrow 8 k+12,8 k+13 \rightarrow 5,8 k+15 \rightarrow 7, \\
& 8 k+17 \rightarrow 9,10 \rightarrow 8 k+10,8 k+11 \rightarrow 3,1,2] \\
& c\left\{1^{4}, 2,8^{8 k+13}\right\}=[0 \rightarrow 8 k+16,5 \rightarrow 8 k+13,8 k+14 \rightarrow 6,7 \rightarrow 8 k+15,4 \rightarrow 8 k+12 \text {, } \\
& 1 \rightarrow 8 k+9,8 k+11 \rightarrow 3,2 \rightarrow 8 k+18,8 k+17]
\end{aligned}
$$




$$
\begin{aligned}
& c\left\{1^{4}, 2,8^{8 k+14}\right\}=[0 \rightarrow 8 k+16,4 \rightarrow 8 k+12,8 k+14 \rightarrow 6,5 \rightarrow 8 k+13,1 \rightarrow 8 k+17,8 k+18, \\
& 8 k+19,7 \rightarrow 8 k+15,3 \rightarrow 8 k+11,8 k+10 \rightarrow 2], \\
& c\left\{1^{4}, 2,8^{8 k+15}\right\}=[0 \rightarrow 8 k+16,3 \rightarrow 8 k+19,8 k+18 \rightarrow 2,1 \rightarrow 8 k+17,8 k+15 \rightarrow 7, \\
& 8 k+20 \rightarrow 4,5 \rightarrow 8 k+13,8 k+14 \rightarrow 6], \\
& c\left\{1^{4}, 2,8^{8 k+16}\right\}=[0 \rightarrow 8 k+16,2 \rightarrow 8 k+18,4 \rightarrow 8 k+20,8 k+19 \rightarrow 3,8 k+17 \rightarrow 1, \\
& 8 k+21 \rightarrow 5,6,7 \rightarrow 8 k+15,8 k+14 \rightarrow 14] \\
& c\left\{1^{4}, 2,8^{8 k+17}\right\}=[0 \rightarrow 8 k+16,1 \rightarrow 8 k+17,2 \rightarrow 8 k+18,3 \rightarrow 8 k+19,4 \rightarrow 8 k+12, \\
& 8 k+13 \rightarrow 5,6 \rightarrow 8 k+14,8 k+15 \rightarrow 7,8 k+22,8 k+20,8 k+21], \\
& c\left\{1^{4}, 2^{2}, 8^{8 k+10}\right\}=[0 \rightarrow 8 k+16,7 \rightarrow 8 k+15,6 \rightarrow 8 k+14,5 \rightarrow 8 k+5,8 k+4 \rightarrow 4, \\
& 2 \rightarrow 8 k+10,8 k+9 \rightarrow 1,3 \rightarrow 8 k+11,8 k+12,8 k+13], \\
& c\left\{1^{4}, 2^{2}, 8^{8 k+11}\right\}=[0 \rightarrow 8 k+16,6 \rightarrow 8 k+14,4 \rightarrow 8 k+12,8 k+13 \rightarrow 5,8 k+15 \rightarrow 7,8 k+17, \\
& 1 \rightarrow 8 k+1,8 k+2 \rightarrow 2,3 \rightarrow 8 k+11,8 k+9,8 k+10], \\
& c\left\{1^{4}, 2^{2}, 8^{8 k+12}\right\}=[0 \rightarrow 8 k+16,5 \rightarrow 8 k+13,8 k+14 \rightarrow 6,7 \rightarrow 8 k+15,4 \rightarrow 8 k+12,1,3, \\
& 2 \rightarrow 8 k+18,8 k+17 \rightarrow 9,11 \rightarrow 8 k+11], \\
& c\left\{1^{4}, 2^{2}, 8^{8 k+13}\right\}=[0 \rightarrow 8 k+16,4 \rightarrow 8 k+12,8 k+14 \rightarrow 6,5 \rightarrow 8 k+13,1 \rightarrow 8 k+17, \\
& 8 k+15 \rightarrow 7,8 k+19,8 k+18,8 k+10,8 k+11 \rightarrow 3,2 \rightarrow 8 k+2] \\
& c\left\{1^{4}, 2^{2}, 8^{8 k+14}\right\}=[0 \rightarrow 8 k+16,3 \rightarrow 8 k+19,8 k+17 \rightarrow 1,2 \rightarrow 8 k+18,8 k+20 \rightarrow 4, \\
& 5 \rightarrow 8 k+13,8 k+14 \rightarrow 6,7 \rightarrow 8 k+15] \\
& c\left\{1^{4}, 2^{2}, 8^{8 k+15}\right\}=[0 \rightarrow 8 k+16,2 \rightarrow 8 k+18,4 \rightarrow 8 k+20,8 k+19 \rightarrow 3,8 k+17 \rightarrow 1, \\
& 8 k+21 \rightarrow 13,14 \rightarrow 8 k+14,8 k+15 \rightarrow 7,5,6], \\
& c\left\{1^{4}, 2^{2}, 8^{8 k+16}\right\}=[0 \rightarrow 8 k+16,1 \rightarrow 8 k+17,2 \rightarrow 8 k+18,8 k+20 \rightarrow 4,8 k+19 \rightarrow 3, \\
& 5 \rightarrow 8 k+5,8 k+6 \rightarrow 6,7 \rightarrow 8 k+15,8 k+14,8 k+22,8 k+21,8 k+13], \\
& c\left\{1^{5}, 2,8^{8 k+10}\right\}=[0 \rightarrow 8 k+16,7 \rightarrow 8 k+15,6 \rightarrow 8 k+14,8 k+13 \rightarrow 5,4 \rightarrow 8 k+12,8 k+11, \\
& 2 \rightarrow 8 k+2,8 k+3 \rightarrow 3,1 \rightarrow 8 k+9,8 k+10], \\
& c\left\{1^{5}, 2,8^{8 k+11}\right\}=[0 \rightarrow 8 k+16,6 \rightarrow 8 k+14,4 \rightarrow 8 k+12,8 k+13 \rightarrow 5,8 k+15 \rightarrow 7,8 k+17, \\
& 1 \rightarrow 8 k+1,8 k+2 \rightarrow 2,3 \rightarrow 8 k+11,8 k+10,8 k+9] \text {, } \\
& c\left\{1^{5}, 2,8^{8 k+12}\right\}=[0 \rightarrow 8 k+16,5 \rightarrow 8 k+13,8 k+14 \rightarrow 6,7 \rightarrow 8 k+15,4 \rightarrow 8 k+12, \\
& 8 k+11 \rightarrow 3,1,2 \rightarrow 8 k+18,8 k+17 \rightarrow 9], \\
& c\left\{1^{5}, 2,8^{8 k+13}\right\}=[0 \rightarrow 8 k+16,4 \rightarrow 8 k+12,8 k+14 \rightarrow 6,5 \rightarrow 8 k+13,1 \rightarrow 8 k+17,8 k+18, \\
& 8 k+19,7 \rightarrow 8 k+15,3,2 \rightarrow 8 k+10,8 k+11 \rightarrow 11], \\
& c\left\{1^{5}, 2,8^{8 k+14}\right\}=[0 \rightarrow 8 k+16,3 \rightarrow 8 k+19,8 k+18 \rightarrow 2,1 \rightarrow 8 k+17,8 k+15 \rightarrow 7, \\
& 6 \rightarrow 8 k+14,8 k+13 \rightarrow 5,4 \rightarrow 8 k+20] \\
& c\left\{1^{5}, 2,8^{8 k+15}\right\}=[0 \rightarrow 8 k+16,2 \rightarrow 8 k+18,4 \rightarrow 8 k+20,8 k+19 \rightarrow 3,8 k+17 \rightarrow 1, \\
& 8 k+21 \rightarrow 13,14 \rightarrow 8 k+14,8 k+15 \rightarrow 7,6,5], \\
& c\left\{1^{5}, 2,8^{8 k+16}\right\}=[0 \rightarrow 8 k+16,1 \rightarrow 8 k+17,2 \rightarrow 8 k+18,3 \rightarrow 8 k+19,4 \rightarrow 8 k+20,8 k+22, \\
& 8 k+21,8 k+13,8 k+14,8 k+15 \rightarrow 7,6 \rightarrow 8 k+6,8 k+5 \rightarrow 5] .
\end{aligned}
$$

\section{Acknowledgments}

The authors thank the anonymous referee whose helpful comments and suggestions improved the presentation of the paper.

\section{References}

[1] M. Buratti, S. Capparelli and A. Del Fra. Cyclic Hamiltonian cycle systems of the $\lambda$-fold complete and cocktail party graphs. European J. Combin., 31:1484-1496, 2010. 
[2] M. Buratti and F. Merola. Hamiltonian cycle systems which are both cyclic and symmetric. To appear in J. Combin. Des., doi:10.1002/jcd.21351.

[3] M. Buratti and F. Merola. Dihedral Hamiltonian Cycle Systems of the Cocktail Party Graph. J. Combin. Des., 21:1-23, 2013.

[4] S. Capparelli and A. Del Fra. Hamiltonian paths in the complete graph with edgelengths 1,2,3. Electron. J. Combin., 17:\#R44, 2010.

[5] J. H. Dinitz and S. R. Janiszewski. On Hamiltonian Paths with Prescribed edge lengths in the Complete Graph. Bull. Inst. Combin. Appl., 57:42-52, 2009.

[6] P. Horak and A. Rosa. On a problem of Marco Buratti. Electron. J. Combin., 16(1):\#R20, 2009.

[7] A. Pasotti and M. A. Pellegrini. A new result on the problem of Buratti, Horak and Rosa. Discrete Math., 319:1-14, 2014.

[8] D. West. http://www.math.uiuc.edu/ west/regs/buratti.html

[9] D. West. Introduction to Graph Theory. Prentice Hall, New Jersey, 1996. 